\title{
Assessment of Soil Moisture Storage In Nigeria Using Climatic Water Budgeting Approach
}

\author{
Musa Oladejo Kehinde \\ Aliyu Tambuwal Umar
}

\begin{abstract}
The estimation of soil moisture storage is fundamental to crop production, hydrological and biological processes. This study assessed soil moisture storage in Nigeria using the Climatic Water Budgeting Approach. Mean monthly air temperature and monthly rainfall data were collected from the archives of the Nigerian Meteorological Agency from 27 weather stations in Nigeria. The data were subjected to Climatic Water Budgeting Approach to compute the monthly soil moisture storage at different locations in Nigeria over two years with contrasting moisture conditions (1983 and 2003). The mean monthly air temperature data were used to estimate the monthly potential evapotranspiration $(P E)$ while the PE in conjunction with the mean monthly rainfall and the soil water holding capacity of $250 \mathrm{~mm}$ were used to calculate the monthly soil moisture storage. The results showed that most locations north of latitude $9^{\circ} \mathrm{N}$ recorded low soil moisture storage below $10 \mathrm{~mm}$ from April to July especially in 1983. The soil moisture storage was high in all the places in January and February due to low potential evapotranspiration and accumulated potential water loss (APWL). Most Places South of latitude $9^{\circ} \mathrm{N}$ recorded higher soil moisture storage between $20 \mathrm{~mm}$ and $100 \mathrm{~mm}$ from January to May compared to their counterparts north of latitude $9^{\circ} \mathrm{N}$ in both 1983 and 2003. The soil moisture storage attained $250 \mathrm{~mm}$ (100\%) from July-October across Nigeria. This study concluded that the soil moisture varies spatially and temporally in Nigeria decreasing from South to North. A paired sample test revealed a significant difference between the soil moisture storage of 2003 and 1983 in Nigeria $(p=.000)$.
\end{abstract}

Key words: Accumulated potential water loss, Potential evapotranspiration, Water holding capacity, Soil moisture storage

Usmanu Danfodiyo University, Department of Geography. *corresponding author e-mail: muskad2008@yahoo.com

Ghana Journal of Geography Vol. 13 (1), 2021 pages 167-202

https://dx.doi.org/10.4314/gjg.v13i1.9 


\section{Introduction}

Soil moisture storage is difficult to define because it means different thing to different disciplines. For example, a farmer's concept of soil moisture is different from that of a weather forecaster or water resource manager (James 1999). In a general term, soil moisture storage refers to the amount of water that is stored in the soil of any particular place at any particular time based on the environmental condition of that particular place and the soil water holding capacity. The major source of moisture in the soil is from the precipitation. Apart from the input from precipitation, the amount of potential evapotranspiration and the soil's ability to hold water plays a significant role in determining the amount of water that will be retained in the soil at any particular time. The moisture storage in the soil at a given time is very crucial for agriculture, especially in places where they so much depend on rainfall for the cultivation of their crops.

The International Water Management Institute (IWMI, 2011) asserts that rain-fed agriculture accounts for $95 \%$ of the total farmed land in Africa. Due to rainfall variability, dry spells and climate change, over reliance on rainfall for cultivation of crops has been a major challenge to farmers over the years. Several studies have demonstrated the effect of soil moisture stress on different crops grown in Nigeria and found out that soil moisture stress result indecline in crop growth and yield (Odunze, Kudi, Daudu, Adeosun, Ayoola, Amapu, Abu, Mand, Ezul, \& Contance, 2010; Fasinmirin \& Olufayo, 2009; Fasinmirin \& Oguntuase, 2008; Shiwachi, Komoda \&Takashi, 2008; Aina, Dixon, \& Akinrinde, 2007). From the foregoing, the estimation of soil moisture storage at different locations and different time will be of immense benefit to the farmers in order to design properly the best time to cultivate their crops for optimum yield. The assessment of soil moisture storage will serve as a very important tool in unfolding the need for irrigation scheme in different parts of Nigeria to augment crop production throughout the year. It will also assist on the quantity of water needed by crop at a particular time. If the farmers have the knowledge about the quantity of water in the soil at different period of the year, they will know whether the water in the soil is sustainable for their crop growth or not. Isoteim (2014) examined the mathematical relationship between the soil moisture and ground water level on a loamy soil in the Niger Delta region of Nigeria. The method made it possible for the people of the region to use the level of water in their shallow wells to determine the moisture status of the soil by measuring the level of water in the wells. Therefore, the method of Isoteim (2014) has become a veritable tool in irrigation scheduling. Soil moisture has a greater influence on the microclimate of any place at a particular time (Jose, Cristiam, Maria, Patricia, and Renan, 2015; Kirien and Sonia, 2015; Syed, Stephane, \& Sylvie, 
2013;; Ahmad and Hashim, 2007 and Andrew, Thomas and Mark, 2002; Oke, 1987). Soil moisture has an effect on the thermal properties of a soil profile as well as conductance and the heat capacity (Varallyay, 2010). Soil moisture and soil thermal properties have a significant influence on seed germination, flowering and fauna activities (Ceri and John, 1991; Maraghni and Neffati, 2010). More water in the soil leads to soil temperature gain or lose (Oke, 1987). The soil moisture in the soil determines the partitioning of the incoming solar radiation into sensible and latent heat (Copernicus, 2013). If there is adequate water in the soil for the incoming radiation to act upon, this will induce convective activities. On the contrary, if there is moisture deficit in the soil, the incoming solar radiation will transform into a sensible heat and make the environment to be very hot thereby bringing physiological discomfort. The recent Climate model by Timbal, Power, Colman, Viviand, and Lirola, (2002) suggested a strong nexus between the soil moisture and the persistence variability of surface temperature and precipitation. They further posited that soil moisture is a significant factor for the accuracy of "inter-annular" predictions regarding the Australian Climate. Soil moisture storage is a veritable tool in controlling the exchange of heat energy between the land surface and the atmosphere through evapotranspiration. In view of this, soil moisture plays an important role in the development of weather pattern and the formation of precipitation (James, 1999)

Nigeria is one of the African countries that rely heavily on rainfall for the cultivation of crops. As such, the knowledge of soil moisture storage will be of tremendous help for efficient crop production. Just like rainfall, irrigation water yields the best result when an optimum quantity of water is applied to a crop. The study of soil moisture storage will not only assist rain-fed agriculture, rather it will serve as a useful guide in the rational scheduling of the application of irrigation water to ensure optimum crop growth (Isoteim, 2014 and Fabura, 2005). The information on soil moisture storage will be useful across varieties of disciplines such as ecology, hydrology, soil science, agronomy and meteorology (Lunt, Hubbard, and Rubin, 2004). If the soil moisture storage at different locations and different period of the year is known, it will serve to define and identify places that suffer from water deficit, a situation that can seriously affect crop production. This will also enables us to determine the soil moisture seasons (Ritter, 2012). Soil moisture storage is very useful information for the determination of the onset and cessation of growing season (Chukwudi, Emma, Ifeanyi and Nwabueze, 2017;Ufoegbune, 2016; Ngetich and Mugendi, 2014; Umar, 2010, Rita, 2007; Ati, Stigter, and Oladepo, 2001; Ati, 1996). When there is adequate moisture in the soil for crop growth, the growing season commences. On the other hand, when there is soil moisture deficiency for crop growth, the growing ceases. 
There is a growing interest in improving the indigenous techniques to assist farmers to adapt to challenges of inadequate water for their crop production. Aboyade, (1990) suggested that efforts be geared towards bridging the gap between the indigenous and the global techniques as a way to facilitate the transfer, acceptance and adoption of modern knowledge system. In the past, fallowing has been described as the best way to improve soil physical, chemical and biological attributes (Hausb and Asawalam, 1998). The long fallow (more than 5 years) is becoming obsolete due to the population pressure on the available land leading to a short fallow (2 to 3 years). With time, this fallow system may go into extinction. As such, scientific research like the soil moisture storage will assist to identify places that are characterized by soil moisture deficit as well as those places that have soil moisture surplus. This information will therefore serve a useful guide in adopting the best technology to boost crop production and water resources management. See Fabura (2005) and Kumar (1995).

The spatial and temporal pattern of water balance indices such as rainfall, potential evapotranspiration, actual evapotranspiration, water deficit, water surplus and runoff have been studied in Nigeria and their monthly and annual values have been mapped out (Ayoade, 1995 and kehinde, 2017. This study aims at bridging the existing gap in literature by assessing the spatio-temporal pattern of soil moisture storage in Nigeria using the Thornthwaite's method of estimating Potential Evapotranspiration (PE) and Climatic Water budgeting Approach.

\section{Methods}

\section{Study Area}

\section{Location}

Nigeria is located approximately between latitude $4^{\circ} \mathrm{N}$ and $14^{\circ} \mathrm{N}$ of the equator and longitude $3^{\circ} \mathrm{E}$ and $15^{\circ} \mathrm{E}$ of the Greenwich meridian. It is bounded in the North by Niger Republic, in the South by Atlantic Ocean and the Gulf of Guinea, in the West by Benin Republic, in the East by Cameroun Republic and in the Northeast by Chad Republic. The total landmass of Nigeria is approximately $923,768 \mathrm{~km}^{2}$ (Iwena, 2000). Nigeria currently comprises thirty-six states and the Federal Capital Territory Abuja. It has 774 Local government areas. See Figure 1 for the location map of Nigeria and the distribution of the weather stations used in this study. 


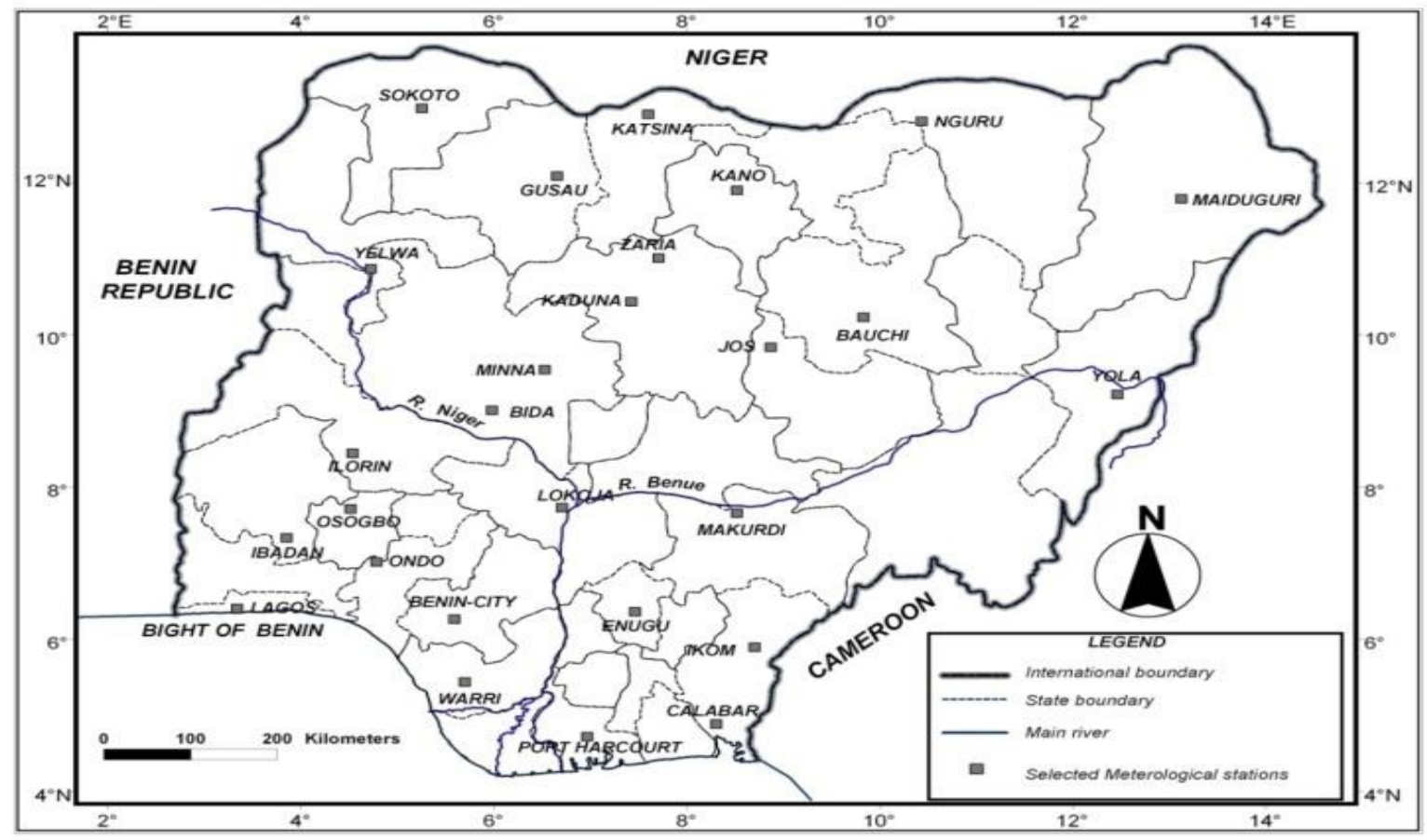

Figure 1. Distribution of Selected Synoptic Meteorological Stations in Nigeria Source: Adapted from Umar (2013)

\section{Thornthwaite Monthly Water Balance Model}

This study followed the Thornthwaite water balance model (Thornthwaite, 1948) which used an accounting procedure to analyze the allocation of water among various components of the hydrologic system. Computation of monthly water balance components of hydrologic cycle were made for a specified location. The inputs into the model are monthly temperature and precipitation. Outputs include monthly potential evapotranspiration and actual evapotranspiration, soil moisture storage, surplus and runoff. The Figure 2shows the component and connectivity of the Thornthwaite water model. The boxes represent the components; the arrows show the data-folw between components 


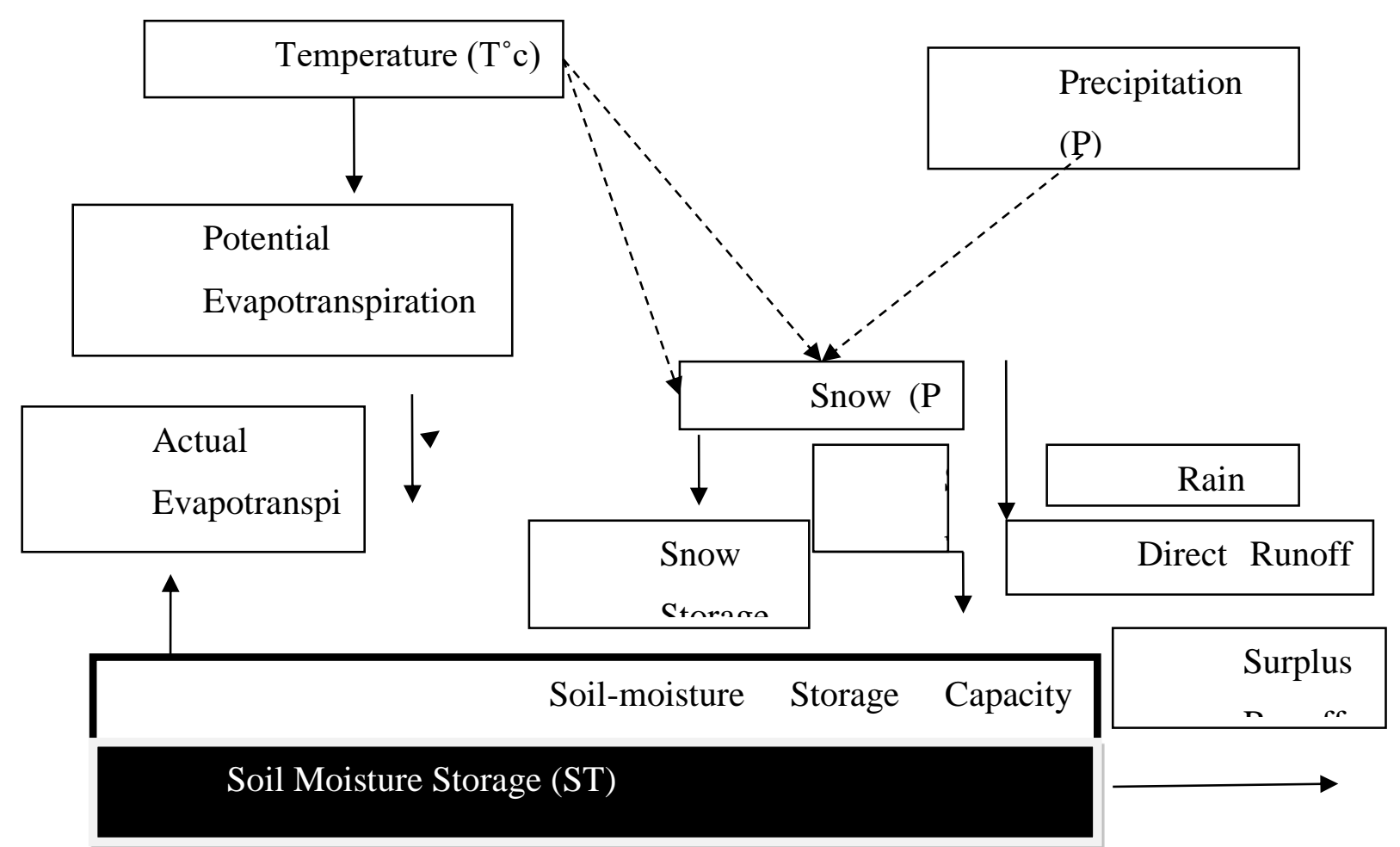

Figure 2: Water Balance Model Schematic

Source: (Thornthwaite Monthly Water Balance Model 2020)

The input data into the model are the monthly air temperature, monthly precipitation (rainfall) and soil water holding capacity. The outputs in the model are potential evapotranspiration (PE or PET), actual soil moisture storage (ST), evapotranspiration (AE), water deficit, water surplus and Runoff.

\section{Types and source of data}

This study used the mean monthly air temperature; the mean monthly rainfall data and the soil water holding capacity of $250 \mathrm{~mm}$ contained in the Thornthwaite's monograph to determine the monthly soil moisture storage. The air temperature and rainfall data were collected from the archives of the Nigerian Meteorological Agency (NIMET) based on twenty-seven weather stations in Nigeria (Figure 1). Two years with contrasting surface moisture condition were selected for this study. The selection of dry year (1983 and wet year (2003) were selected consequence upon the study of dry and wet years by Umar (2013) This is to see whether there is any significant different between the soil moisture storage of the year 1983 and 2003 in Nigeria. 


\section{Method of Data Analysis and Presentation}

The computation of soil moisture storage for different locations in Nigeria was done via the Thornthwaite's water balance model, which deals with computation of a water balance sheet. The balance sheet requires inputs of monthly estimated PE values and monthly precipitation to arrive at the monthly soil moisture storage. The water balance sheet begins with the estimation of monthly PE thus:

\section{Calculation of Potential Evapotranspiration}

The air temperature data collected from the archives of the Nigerian Meteorological Agency was subjected to the Thornthwaite, (1948) approach to calculate the monthly potential evapotranspiration. Thornthwaite (1948) developed empirical equation for estimating potential evapotranspiration and this is given as:

$$
\begin{aligned}
& E T_{0}=16 \times(10 \mathrm{i} / \mathrm{I}){ }^{\mathrm{a}}(\mathrm{N} / 12)(1 / 30) \\
& \mathrm{I}=\sum_{I=1}^{12}(T i / 5) 1.514
\end{aligned}
$$

Where,

$T i=$ the mean monthly temperature in $\left({ }^{\circ} \mathrm{C}\right)$.

$N=$ the monthly Sunshine hours.

$I=$ the heat index.

The equation has been packaged into a monograph for easy estimation of PE. Therefore, the estimation of PE was done with the aid of Thornthwaite's monograph. The monograph contained the values of air temperature in degree Celsius with their corresponding values of monthly heat index, values of unadjusted daily potential evapotranspiration $(\mathrm{mm})$ for different mean temperature $\left({ }^{\circ} \mathrm{C}\right)$ and annual heat index (I) values. Thornthwaite's monograph also contained mean possible monthly duration of sunlight in the northern hemisphere and southern hemisphere respectively.

After estimating the monthly PE for each location across Nigeria, the values in conjunction with the monthly rainfall were subjected to the water balance sheet to determine the soil moisture storage.The water balance sheet was computed for each location and it involves the following the steps: 
Step 1: P-PE: This is the difference between the monthly precipitation and the monthly potential evapotranspiration.P is the monthly precipitation in a year while $\mathbf{P E}$ is the monthly estimated potential evapotranspiration.

Step 2: APWL: This is the accumulated potential water loss. This only arises when PE $>\mathrm{P}$.* If $\mathrm{PE}>\mathrm{P}$, then APWL is a cumulative value of $(\mathrm{P}-\mathrm{PE}) . *$ If $\mathrm{PE}<\mathrm{P}$, then no APWL.

Step 3: Thisinvolvesdetermining the exactmonthly soil moisture storage (ST)with the aid of Thornthwaite's monograph of water holding capacity of $250 \mathrm{~mm}$. This is obtained in the monograph but only in months where the APWL occurs. Other months without APWL, ST = preceding ST + P-PE.

This estimation of soil moisture storage was done for each of the twenty-seven (27) weather stations selected across Nigeria for the study and the presentation of results was made using tables, spatial maps and charts for better understanding. A paired $t$ test was used to test whether there is any significant difference between the soil moisture storage of 2003 and 1983 in Nigeria.

\section{Result}

\section{Spatial variation of soil moisture storage in Nigeria}

It is very important to note that the monthly potential evapotranspiration (PE), monthly rainfall and monthly-accumulated potential water loss (APWL) are perquisite for determining the monthly values of soil moisture storage (ST). Therefore, the results of each of these parameters are presented in different tables for better understanding the study. Table 1ashowed the computed monthly values of PEfor the different locationsacross Nigeria for 1983. The table revealed that the potential evapotranspiration values are higher during from the months of April to October where many of the locations recorded monthly PE values between $150 \mathrm{~mm}$ and $192 \mathrm{~mm}$. When the PE is high, the APWL will also be high. It further implies the higher the APWL, the higher lower the soil moisture storage. On the contrary, the monthly values of PE were low between December and January where most of the locations north of latitude $10^{\circ} \mathrm{N}$ recorded PE as low as between $25 \mathrm{~mm}$ and $100 \mathrm{~mm}$ in January. 
Table 1a Monthly Potential evapotranspiration for 1983 in Nigeria

\begin{tabular}{|c|c|c|c|c|c|c|c|c|c|c|c|c|c|}
\hline Station & Lat. & Jan. & Feb. & Mar. & Apr & May & June & Jul. & Aug. & Sep. & Oct. & Nov. & Dec. \\
\hline Katsina & 13.01 & 37 & 181 & 182 & 192 & 191 & 190 & 179 & 172 & 180 & 187 & 182 & 173 \\
\hline Sokoto & 13.01 & 192 & 192 & 192 & 169 & 159 & 168 & 184 & 181 & 165 & 185 & 185 & 159 \\
\hline Nguru & 12.53 & 24 & 175 & 169 & 192 & 188 & 190 & 182 & 174 & 181 & 185 & 176 & 153 \\
\hline Gusau & 12.1 & 66 & 190 & 190 & 189 & 192 & 188 & 179 & 169 & 179 & 189 & 191 & 184 \\
\hline Kano & 12.03 & 28 & 157 & 162 & 190 & 191 & 178 & 164 & 156 & 167 & 177 & 167 & 143 \\
\hline Zaria & 11.6 & 54 & 182 & 183 & 192 & 190 & 172 & 157 & 152 & 159 & 179 & 179 & 171 \\
\hline Maiduguri & 11.51 & 30 & 170 & 173 & 192 & 191 & 185 & 173 & 171 & 180 & 188 & 179 & 164 \\
\hline Bauchi & 10.7 & 43 & 170 & 171 & 190 & 185 & 159 & 147 & 144 & 158 & 171 & 173 & 160 \\
\hline Yelwa & 10.53 & 190 & 183 & 173 & 192 & 191 & 182 & 174 & 165 & 175 & 191 & 192 & 191 \\
\hline Kaduna & 10.36 & 117 & 163 & 182 & 180 & 157 & 152 & 117 & 143 & 127 & 157 & 166 & 119 \\
\hline Jos & 9.52 & 51 & 141 & 142 & 160 & 106 & 83 & 74 & 67 & 97 & 125 & 132 & 115 \\
\hline Minna & 9.37 & 173 & 192 & 192 & 192 & 188 & 167 & 156 & 150 & 158 & 182 & 188 & 187 \\
\hline Yola & 9.14 & 90 & 188 & 190 & 191 & 190 & 170 & 155 & 157 & 162 & 175 & 181 & 180 \\
\hline Bida & 9.06 & 176 & 192 & 192 & 192 & 189 & 173 & 165 & 158 & 165 & 183 & 189 & 187 \\
\hline Ilorin & 8.29 & 120 & 174 & 179 & 176 & 165 & 145 & 110 & 103 & 140 & 160 & 170 & 156 \\
\hline Lokoja & 7.47 & 153 & 185 & 188 & 186 & 178 & 157 & 153 & 148 & 156 & 172 & 179 & 174 \\
\hline Oshogbo & 7.47 & 181 & 190 & 191 & 187 & 173 & 153 & 147 & 107 & 155 & 172 & 179 & 172 \\
\hline Makurdi & 7.44 & 176 & 192 & 192 & 192 & 183 & 165 & 157 & 160 & 164 & 178 & 186 & 187 \\
\hline Ibadan & 7.26 & 176 & 190 & 181 & 173 & 159 & 142 & 11 & 95 & 123 & 155 & 88 & 162 \\
\hline Ondo & 7.06 & 182 & 188 & 191 & 183 & 169 & 145 & 140 & 93 & 144 & 164 & 173 & 169 \\
\hline Enugu & 6.28 & 152 & 180 & 184 & 183 & 166 & 151 & 144 & 119 & 147 & 159 & 171 & 168 \\
\hline Lag os & 6.27 & 156 & 171 & 176 & 174 & 163 & 142 & 126 & 112 & 141 & 157 & 163 & 157 \\
\hline Benin & 6.19 & 183 & 189 & 189 & 182 & 177 & 152 & 150 & 103 & 146 & 169 & 173 & 176 \\
\hline Ikom & 5.58 & 171 & 190 & 192 & 187 & 177 & 163 & 154 & 146 & 160 & 171 & 177 & 177 \\
\hline Warri & 5.31 & 157 & 172 & 176 & 175 & 163 & 147 & 120 & 114 & 122 & 155 & 161 & 156 \\
\hline Calabar & 4.58 & 175 & 186 & 188 & 178 & 164 & 147 & 140 & 102 & 143 & 151 & 165 & 167 \\
\hline Port/H & 4.51 & 157 & 177 & 181 & 167 & 159 & 146 & 122 & 113 & 124 & 146 & 157 & 156 \\
\hline
\end{tabular}

Source: Author's Computation (2017)

The result in Table $1 \mathrm{~b}$ contained the monthly rainfall recorded at different locations in Nigeria for the year 1983 . The table revealed that most of the places north of latitude $10^{\circ} \mathrm{Nrecorded}$ 
little or no rainfall from January to May and October to December compared to other locations south of latitude $10^{\circ} \mathrm{N}$. Significant rainfall between $50 \mathrm{~mm}$ and $250 \mathrm{~mm}$ were recorded in only four months (June to September) in locations north of latitude $10^{\circ} \mathrm{N}$ while most of the places south of latitude $9^{\circ} \mathrm{N}$ recorded significant rainfall between $50 \mathrm{~mm}$ and $300 \mathrm{~mm}$ at least 8 months (March to October). The amount of rainfall and PE at different locations contribute immensely to the amount of ST.

The Accumulated Potential Water Loss (APWL) for 1983 in Table 1c is a strong prerequisite for the determinant of the monthly values of soil moisture storage every monthly PE. The result in the Table $1 \mathrm{c}$ showed that the monthly APWL are higher in most places north of latitude $10^{\circ} \mathrm{N}$ in virtually all the months compared to their counterparts in the south of latitude $10^{\circ} \mathrm{N}$. This implies that places north of latitude $10^{\circ} \mathrm{N}$ with higher APWL will experience low soil moisture storage compared to those locations south of latitude $10^{\circ} \mathrm{N}$. The higher the APWL the lower the Soil moisture storage (ST) will be. This assertion is in line with the finding of Kehinde,Umar and Bulama(2019), which examined the relationship between APWL and soil moisture storage in semi-Arid Region of Nigeria and found that there is a significant negative relationship between the soil moisture storage and the APWL in all the place in Nigeria.

Tables 2a showed that the computed monthly PE for 2003 varies across different locations in Nigeria. It could be seen from the table that the monthly PE is higher across different locations in 2003 compared to that of 1983. The monthly PE values are higher (between $150 \mathrm{~mm}$ and $192 \mathrm{~mm}$ ) in many places north of latitude $9^{\circ} \mathrm{N}$ especially from March to August compared otter months. In contrast, the monthly PE values ranges from $140 \mathrm{~mm}$ to $190 \mathrm{~mm}$ in many locations south of latitude $9^{\circ} \mathrm{N}$ from March to October. This implies that rate of amount of PE is increases from south to north in Nigeria.

The result in Table $2 b$ contained the monthly amount of rainfall for 2003 across different locations in Nigeria. It could be observed from the table that the monthly rainfall varies $4 \mathrm{~mm}$ and $500 \mathrm{~m}$ between April and September. The table also revealed that amount of rainfall decreases from the south towards the north.

The APWLas a prerequisite for determining the monthly values of the soil moisture storage for the year 2003 is depicted in Table 2c. The result shows that the monthly APWL is high from January to June across different locations north of latitude $9^{\circ} \mathrm{N}$ compared to locations south of $9^{\circ} \mathrm{N}$. The high-accumulated potential water loss in many places across Nigeria is an indication of low soil moisture storage. 
Table 1b Monthly Rainfall for 1983 in Nigeria Values (mm)

\begin{tabular}{|c|c|c|c|c|c|c|c|c|c|c|c|c|c|}
\hline Station & Lat. & Jan & Feb & Mar. & Apr & May & June & Jul & Aug. & Sep. & Oct. & Nov & Dec. \\
\hline Katsina & 13.01 & 0 & 0 & 0.1 & 0 & 0.4 & 90 & 109 & 101 & 101 & 0 & 0 & 0 \\
\hline Sokoto & 13.01 & 0 & 0 & 0 & 0 & 45 & 154 & 229 & 128 & 62 & 0 & 0 & 0 \\
\hline Nguru & 12.53 & 0 & 0 & 2 & 0 & 0 & 56 & 42 & 89 & 38 & 0 & 0 & 0 \\
\hline Gusau & 12.1 & 0 & 0 & 7 & 0 & 57 & 107 & 229 & 287 & 161 & 0 & 0 & 0 \\
\hline Kano & 12.03 & 0 & 0 & 0 & 0 & 34 & 40 & 92 & 231 & 67 & 0 & 0 & 0 \\
\hline Zaria & 11.6 & 0 & 0 & 0 & 0 & 63 & 129 & 133 & 287 & 71 & 0.4 & 0 & 0 \\
\hline Maiduguri & 11.51 & 0 & 0 & 8 & 0 & 2 & 40 & 71 & 100 & 36 & 0 & 0 & 0 \\
\hline Bauchi & 10.7 & 0 & 0 & 13 & 0 & 95 & 124 & 227 & 184 & 136 & 0 & 0 & 0 \\
\hline Yelwa & 10.53 & 0 & 0 & 0 & 11 & 124 & 59 & 129 & 137 & 103 & 0 & 0 & 0 \\
\hline Kaduna & 10.36 & 0 & 0 & 18 & 0 & 135 & 180 & 113 & 204 & 235 & 0 & 0 & 0 \\
\hline Jos & 9.52 & 0 & 0 & 21 & 0 & 150 & 250 & 327 & 359 & 69 & 0 & 0 & 0 \\
\hline Minna & 9.37 & 0 & 0 & 0 & 12 & 97 & 97 & 159 & 300 & 229 & 34 & 0 & 0 \\
\hline Yola & 9.14 & 0 & 0 & 0 & 0 & 99 & 107 & 223 & 209 & 89 & 11 & 0 & 0 \\
\hline Bida & 9.06 & 0 & 0 & 0 & 8 & 54 & 121 & 149 & 170 & 244 & 2 & 0 & 0 \\
\hline Ilorin & 8.29 & 0 & 9 & 15 & 92 & 169 & 146 & 92 & 216 & 228 & 34 & 2 & 149 \\
\hline Lokoja & 7.47 & 0 & 0 & 48 & 62 & 100 & 193 & 120 & 191 & 125 & 15 & 0 & 0 \\
\hline Oshogbo & 7.47 & 0 & 37 & 23 & 81 & 252 & 178 & 84 & 62 & 220 & 74 & 26 & 52 \\
\hline Makurdi & 7.44 & 0 & 0 & 1.2 & 3 & 193 & 214 & 232 & 158 & 128 & 0.8 & 0 & 0 \\
\hline Ibadan & 7.26 & 0 & 17 & 0 & 85 & 241 & 200 & 47 & 46 & 93 & 70 & 17 & 42 \\
\hline Ondo & 7.06 & 0 & 18 & 35 & 0 & 155 & 283 & 129 & 45 & 312 & 80 & 47 & 22 \\
\hline Enugu & 6.28 & 0 & 0 & 4 & 42 & 152 & 101 & 251 & 100 & 241 & 24 & 3 & 0 \\
\hline Lagos & 6.27 & 0 & 0 & 48 & 58 & 221 & 326 & 51 & 19 & 77 & 37 & 33 & 23 \\
\hline Benin & 6.19 & 0 & 49 & 39 & 77 & 267 & 278 & 167 & 127 & 425 & 148 & 30 & 23 \\
\hline Ikom & 5.58 & 0 & 189 & 27 & 157 & 279 & 293 & 318 & 360 & 361 & 61 & 62 & 3 \\
\hline Warri & 5.31 & 0 & 49 & 6 & 128 & 352 & 407 & 351 & 103 & 671 & 177 & 88 & 191 \\
\hline Calabar & 4.58 & 0 & 14 & 16 & 138 & 290 & 504 & 291 & 342 & 293 & 229 & 114 & 67 \\
\hline Port/H & 4.51 & 0 & 0 & 33 & 114 & 141 & 184 & 311 & 155 & 324 & 305 & 20 & 46 \\
\hline
\end{tabular}

Source: Author's Computation (2017) 
Table 1c Monthly Accumulated Potential Water Loss (APWL) (mm) in Nigeria for 1983

\begin{tabular}{|c|c|c|c|c|c|c|c|c|c|c|c|c|c|}
\hline Station & Lat & Jan & Feb & Mar & Apr & May & Jun & Jul & Aug & Sept & Oct & Nov. & Dec \\
\hline Minna & 9.37 & -360 & -525 & -744 & -924 & -1015 & -1155 & 0 & 0 & 0 & -148 & -336 & -523 \\
\hline Zaria & 11.6 & -225 & -407 & -590 & -782 & -909 & -952 & -976 & 0 & -88 & -267 & -446 & 1617 \\
\hline Yelwa & 10.53 & -381 & -564 & -737 & -918 & -976 & -1099 & -1144 & -1172 & -1244 & -1435 & -1627 & -1818 \\
\hline Kano & 12.03 & -171 & -328 & -490 & -680 & -837 & -975 & -1047 & 0 & -100 & -277 & -444 & -587 \\
\hline Maid & 11.51 & -194 & -364 & -529 & -721 & -910 & -1055 & -1157 & -1228 & -1372 & -1560 & -1739 & -1903 \\
\hline Kadu & 10.36 & -236 & -399 & -563 & -743 & -765 & 0 & -4 & 0 & 0 & -157 & -323 & -442 \\
\hline Yola & 9.14 & -270 & -458 & -648 & -839 & -930 & -993 & 0 & 0 & -73 & -237 & -418 & -598 \\
\hline Bauch & 10.7 & -203 & -373 & -531 & 721 & -811 & -846 & 0 & 0 & -22 & -193 & -366 & -526 \\
\hline Nguru & 12.53 & -177 & -352 & -519 & -711 & -899 & -1033 & -1173 & -1288 & -1401 & 1586 & -1762 & -1915 \\
\hline Katsin & 13.01 & -210 & -391 & -573 & -765 & -956 & -1048 & -1118 & 1189 & -1268 & -1455 & -1637 & -1810 \\
\hline Jos & 9.52 & -166 & -307 & -428 & -588 & 0 & 0 & 0 & 0 & -28 & -153 & -285 & -400 \\
\hline Enugu & 6.28 & 1320 & -500 & -680 & -821 & -835 & -885 & 0 & -19 & 0 & -135 & -303 & -471 \\
\hline Benin & 6.19 & -336 & -476 & -626 & -731 & 0 & 0 & 0 & 0 & 0 & -21 & -164 & -317 \\
\hline Lokoja & 7.47 & -32 & -512 & -652 & -776 & -854 & 0 & -33 & 0 & -31 & -188 & -367 & -541 \\
\hline Makurdi & 7.44 & -363 & -555 & -746 & -935 & 0 & 0 & 0 & -2 & -38 & -215 & -401 & -588 \\
\hline Ondo & 7.6 & -329 & -499 & -655 & -838 & -852 & 0 & -11 & -55 & 0 & -84 & -210 & -357 \\
\hline Oshogbo & 7.47 & -301 & -454 & -622 & -728 & 0 & 0 & -63 & -108 & 0 & -98 & -251 & -371 \\
\hline Warri & 5.31 & -122 & -245 & -415 & -462 & 0 & 0 & 0 & -11 & 0 & 0 & -73 & 0 \\
\hline Pot/H & 4.51 & -267 & -444 & -592 & -645 & -663 & 0 & 0 & 0 & 0 & 0 & -137 & -247 \\
\hline Soot & 13.01 & -351 & -543 & -735 & -904 & -1018 & 1032 & 0 & -53 & -156 & -341 & -526 & -685 \\
\hline Ilorin & 8.25 & -127 & -292 & -456 & -540 & 0 & 0 & -18 & 0 & 0 & -126 & -294 & -301 \\
\hline Bida & 9.06 & -363 & -555 & -747 & 931 & -932 & -984 & -1000 & 0 & 0 & -181 & -370 & -557 \\
\hline Iba & 7.27 & -296 & -469 & -650 & -738 & 0 & 0 & -64 & 113 & -143 & -228 & -299 & -419 \\
\hline Lagos & 6.27 & -290 & -461 & -589 & -705 & 0 & 0 & -75 & -168 & -232 & -352 & -482 & -616 \\
\hline Calab & 4.58 & -275 & -447 & -609 & -649 & 0 & 0 & 0 & 0 & 0 & 0 & -51 & -151 \\
\hline Ikom & 5.58 & -345 & -346 & -510 & -540 & 0 & 0 & 0 & 0 & 0 & -110 & -225 & -399 \\
\hline Gusa & 12.1 & -250 & -440 & -623 & -812 & -947 & -1028 & 0 & 0 & -18 & -207 & -398 & -582 \\
\hline
\end{tabular}

Source: Author's Computation (2017) 
Table 2a. Monthly Potential Evapotranspiration (mm) for 2003 in Nigeria

\begin{tabular}{|c|c|c|c|c|c|c|c|c|c|c|c|c|c|}
\hline Station & Lat & Jan. & Feb. & Mar. & Apr & May & June & Jul. & Aug. & Sep. & Oct. & Nov. & Dec. \\
\hline Katsina & 13.01 & 171 & 188 & 189 & 191 & 192 & 186 & 173 & 164 & 170 & 189 & 186 & 163 \\
\hline Sokoto & 13.01 & 161 & 183 & 186 & 192 & 192 & 181 & 167 & 151 & 165 & 185 & 185 & 159 \\
\hline Nguru & 12.53 & 148 & 172 & 182 & 192 & 192 & 180 & 171 & 163 & 176 & 188 & 181 & 142 \\
\hline Gusau & 12.1 & 183 & 192 & 192 & 192 & 192 & 159 & 180 & 170 & 180 & 190 & 191 & 184 \\
\hline Kano & 12.03 & 111 & 166 & 175 & 189 & 190 & 167 & 153 & 146 & 159 & 178 & 172 & 105 \\
\hline Zaria & 11.6 & 171 & 187 & 188 & 190 & 189 & 166 & 157 & 145 & 159 & 176 & 178 & 162 \\
\hline Maiduguri & 11.51 & 156 & 177 & 185 & 192 & 192 & 181 & 171 & 153 & 170 & 191 & 182 & 151 \\
\hline Bauchi & 10.7 & 153 & 189 & 190 & 187 & 188 & 164 & 159 & 153 & 170 & 175 & 149 & 169 \\
\hline Yelwa & 10.53 & 167 & 192 & 190 & 192 & 186 & 180 & 167 & 155 & 171 & 181 & 173 & 90 \\
\hline Kaduna & 10.36 & 157 & 178 & 181 & 176 & 175 & 147 & 124 & 114 & 120 & 160 & 168 & 148 \\
\hline Jos & 9.52 & 122 & 147 & 157 & 125 & 117 & 80 & 71 & 69 & 84 & 117 & 129 & 110 \\
\hline Minna & 9.37 & 189 & 192 & 192 & 191 & 189 & 164 & 162 & 158 & 160 & 173 & 188 & 187 \\
\hline Yola & 9.14 & 175 & 189 & 191 & 192 & 191 & 183 & 159 & 155 & 157 & 170 & 181 & 169 \\
\hline Bida & 9.06 & 190 & 192 & 192 & 192 & 189 & 169 & 171 & 167 & 163 & 181 & 189 & 189 \\
\hline Ilorin & 8.29 & 168 & 178 & 181 & 171 & 169 & 147 & 121 & 118 & 143 & 157 & 167 & 167 \\
\hline Lokoja & 7.47 & 174 & 185 & 187 & 181 & 180 & 156 & 153 & 169 & 165 & 179 & 185 & 187 \\
\hline Oshogbo & 7.47 & 184 & 187 & 189 & 176 & 177 & 160 & 149 & 149 & 156 & 168 & 177 & 186 \\
\hline Makurdi & 7.44 & 190 & 192 & 192 & 190 & 190 & 171 & 168 & 163 & 163 & 177 & 186 & 188 \\
\hline Ibadan & 7.26 & 164 & 173 & 178 & 165 & 165 & 146 & 121 & 119 & 143 & 158 & 155 & 162 \\
\hline Ondo & 7.06 & 178 & 187 & 187 & 172 & 172 & 158 & 82 & 141 & 149 & 171 & 174 & 182 \\
\hline Enugu & 6.28 & 174 & 181 & 182 & 174 & 169 & 149 & 151 & 151 & 147 & 162 & 170 & 167 \\
\hline Lagos & 6.27 & 162 & 172 & 174 & 168 & 170 & 146 & 146 & 146 & 149 & 160 & 163 & 169 \\
\hline Benin & 6.19 & 181 & 187 & 183 & 176 & 173 & 155 & 150 & 145 & 141 & 167 & 177 & 178 \\
\hline Ikom & 5.58 & 181 & 189 & 190 & 179 & 175 & 161 & 157 & 149 & 157 & 170 & 173 & 173 \\
\hline Warri & 5.31 & 165 & 172 & 176 & 166 & 160 & 150 & 177 & 123 & 147 & 158 & 165 & 162 \\
\hline Calabar & 4.58 & 179 & 182 & 180 & 172 & 168 & 160 & 155 & 144 & 153 & 157 & 165 & 172 \\
\hline Port/ H & 4.51 & 179 & 186 & 181 & 173 & 164 & 152 & 149 & 147 & 150 & 160 & 166 & 175 \\
\hline
\end{tabular}

Source: Author's Computation (2017) 
Ghana Journal of Geography Vol. 13 (1), 2021 pages 167-202

Table 2b. Monthly Rainfall (mm) for 2003 in Nigeria.

\begin{tabular}{|c|c|c|c|c|c|c|c|c|c|c|c|c|c|}
\hline Station & Lat. & Jan. & Feb. & Mar. & Apr & May & June & Jul. & Aug. & Sep. & Oct. & Nov. & Dec. \\
\hline Katsina & 13.01 & 0 & 0 & 0 & 4 & 13 & 46 & 115 & 276 & 76 & 16 & 0 & 0 \\
\hline Soot & 13.01 & 0 & 0 & 0 & 9 & 18 & 71 & 288 & 300 & 108 & 7 & 0 & 0 \\
\hline Nguru & 12.53 & 0 & 0 & 0 & 0 & 2 & 2 & 97 & 188 & 41 & 0 & 0 & 0 \\
\hline Gusau & 12.1 & 0 & 1 & 1 & 12 & 151 & 176 & 271 & 437 & 199 & 18 & 0 & 0 \\
\hline Kano & 12.03 & 0 & 0 & 0 & 10 & 67 & 247 & 338 & 458 & 233 & 13 & 0 & 0 \\
\hline Zaria & 11.6 & 0 & 0 & 0 & 56 & 93 & 72 & 254 & 407 & 238 & 63 & 0 & 0 \\
\hline Maiduguri & 11.51 & 0 & 0 & 0 & 4 & 13 & 89 & 203 & 232 & 79 & 15 & 0 & 0 \\
\hline Bauchi & 10.7 & 0 & 0 & 0 & 31 & 26 & 309 & 134 & 263 & 173 & 31 & 0 & 0 \\
\hline Yelwa & 10.53 & 0 & 0 & 24 & 18 & 199 & 98 & 126 & 397 & 173 & 45 & 0 & 0 \\
\hline Kaduna & 10.36 & 0 & 0 & 28 & 76 & 84 & 152 & 399 & 426 & 397 & 71 & 0 & 0 \\
\hline Jos & 9.52 & 0 & 0 & 0 & 200 & 156 & 184 & 161 & 283 & 258 & 36 & 2 & 0 \\
\hline Minna & 9.37 & 0 & 6 & 0 & 17 & 98 & 213 & 117 & 192 & 187 & 192 & 2 & 0 \\
\hline Yola & 9.14 & 0 & 0 & 0 & 7 & 73 & 113 & 175 & 236 & 262 & 61 & 0 & 0 \\
\hline Bida & 9.06 & 0 & 0 & 0 & 9 & 146 & 154 & 147 & 179 & 144 & 81 & 31 & 0 \\
\hline Ilorin & 8.29 & 0 & 0 & 25 & 82 & 96 & 371 & 84 & 81 & 400 & 0 & 18 & 0 \\
\hline Lokoja & 7.47 & 0 & 0 & 0 & 0 & 93 & 181 & 271 & 53 & 164 & 147 & 15 & 0 \\
\hline Oshogbo & 7.47 & 21 & 0 & 15 & 176 & 99 & 146 & 105 & 93 & 287 & 266 & 85 & 0 \\
\hline Makurdi & 7.44 & 0 & 0 & 0 & 56 & 31 & 200 & 119 & 133 & 136 & 40 & 34 & 0 \\
\hline Ibadan & 7.26 & 25 & 82 & 4 & 184 & 91 & 315 & 228 & 58 & 215 & 206 & 52 & 0 \\
\hline Ondo & 7.06 & 16 & 13 & 54 & 289 & 81 & 192 & 61 & 81 & 557 & 184 & 123 & 0 \\
\hline Enugu & 6.28 & 1 & 3 & 8 & 187 & 306 & 222 & 285 & 137 & 296 & 269 & 22 & 33 \\
\hline Lagos & 6.27 & 174 & 53 & 79 & 308 & 156 & 321 & 70 & 19 & 185 & 141 & 185 & 0 \\
\hline Benin & 6.19 & 0 & 14 & 109 & 233 & 189 & 331 & 124 & 263 & 173 & 237 & 160 & 0 \\
\hline Ikom & 5.58 & 8 & 0 & 65 & 151 & 144 & 304 & 363 & 224 & 315 & 410 & 50 & 7 \\
\hline Warri & 5.31 & 54 & 6 & 153 & 239 & 90 & 234 & 351 & 199 & 436 & 365 & 115 & 10 \\
\hline Calabar & 4.58 & 27 & 103 & 197 & 283 & 318 & 202 & 282 & 381 & 309 & 186 & 149 & 3 \\
\hline Port/H & 4.51 & 17 & 86 & 93 & 169 & 166 & 242 & 433 & 200 & 535 & 204 & 99 & 4 \\
\hline
\end{tabular}

Source: Author's Computation (2017) 
Table 2c. Monthly Accumulated Potential Water Loss in Nigeria for 2003

\begin{tabular}{|c|c|c|c|c|c|c|c|c|c|c|c|c|c|}
\hline Station & Lat & Jan & Feb & Ma & Apr & May & Jun & Jul & Aug & Sep & Oct & Nov & Dec \\
\hline Minna & 9.37 & -376 & -562 & -754 & -928 & -1019 & 0 & -45 & 0 & 0 & 0 & -186 & -373 \\
\hline Zaria & 11.6 & -333 & -520 & -708 & -842 & -938 & -1032 & 0 & 0 & 0 & -113 & -291 & -453 \\
\hline Yelwa & 10.53 & -257 & -449 & -615 & -789 & 0 & -82 & -123 & 0 & 0 & -136 & -309 & -399 \\
\hline Kano & 12.03 & -216 & -382 & -557 & -736 & -859 & 0 & 0 & 0 & 0 & -165 & -337 & -442 \\
\hline Maidu, & 11.51 & -307 & -484 & -669 & -857 & -1036 & -1128 & 0 & 0 & -91 & -287 & -469 & -620 \\
\hline $\mathrm{Kad}$ & 10.36 & -305 & -483 & -636 & -736 & -827 & 0 & 0 & 0 & 0 & -89 & -257 & -405 \\
\hline Yola & 9.14 & -344 & -533 & -724 & -909 & -1027 & -1097 & 0 & 0 & 0 & -109 & -290 & -350 \\
\hline Bauc & 10.7 & -322 & -511 & -701 & -857 & -1019 & 0 & -25 & 0 & 0 & -144 & -293 & -462 \\
\hline Nguru & 12.53 & -290 & -462 & -644 & -836 & -1026 & -1204 & -1278 & 0 & -135 & -323 & -504 & -646 \\
\hline Kats & 13.01 & -334 & -522 & -711 & -898 & -1077 & -1217 & -1275 & 0 & -94 & -267 & -453 & -616 \\
\hline Jos & 9.52 & -232 & -379 & -536 & 0 & 0 & 0 & 0 & 0 & 0 & -81 & -208 & -318 \\
\hline Enu & 6.28 & -307 & -485 & -659 & 0 & 0 & 0 & 0 & -14 & 0 & 0 & -148 & -282 \\
\hline Benin & 6.19 & -198 & -371 & -445 & 0 & 0 & 0 & -26 & 0 & 0 & 0 & -17 & -195 \\
\hline Lok & 7.47 & -361 & -546 & -733 & -914 & -100 & 0 & 0 & -116 & -117 & -149 & -319 & -506 \\
\hline Mak & 7.44 & -378 & -570 & -762 & -896 & -1055 & 0 & -49 & -79 & -106 & -243 & -395 & -583 \\
\hline Ondo & 7.6 & -344 & -518 & -651 & 0 & -91 & 0 & -21 & -81 & 0 & 0 & -51 & -233 \\
\hline Osho & 7.47 & -349 & -536 & -710 & 0 & -78 & -92 & -136 & -190 & 0 & 0 & -92 & -278 \\
\hline Warri & 5.31 & -263 & -429 & -452 & 0 & -70 & 0 & 0 & 0 & 0 & 0 & -50 & -202 \\
\hline Port/H & 4.51 & -333 & -433 & -521 & -525 & 0 & 0 & 0 & 0 & 0 & 0 & -67 & -238 \\
\hline Sokoto & 13.01 & -320 & -503 & -689 & -872 & -1046 & -1156 & 0 & 0 & -57 & -235 & -420 & -579 \\
\hline Ilorin & 8.25 & -335 & -513 & -669 & -758 & -831 & 0 & -37 & -74 & 0 & -157 & -309 & -473 \\
\hline Bida & 9.06 & -379 & -571 & -763 & -946 & -989 & -1004 & -1028 & 0 & -19 & -119 & -277 & -466 \\
\hline Ibadan & 7.27 & -301 & -392 & -566 & 0 & -74 & 0 & 0 & -61 & 0 & 0 & -103 & -265 \\
\hline Lagos & 6.27 & -157 & -276 & -371 & 0 & -14 & 0 & -76 & -203 & 0 & -19 & 0 & -169 \\
\hline Calabar & 4.58 & -321 & -400 & 0 & 0 & 0 & 0 & 0 & 0 & 0 & 0 & -16 & -185 \\
\hline Ikom & 5.58 & -339 & -528 & -653 & -681 & -712 & 0 & 0 & 0 & 0 & 0 & -123 & -289 \\
\hline Gusau & 12.1 & -367 & -558 & -749 & -929 & -970 & 0 & 0 & 0 & 0 & -172 & -363 & -547 \\
\hline
\end{tabular}

Source: Author's Computation (2017) 
The monthly spatial patterns of soil moisture storage in Nigeria for 1983 and 2003 are contained in Figure 3. The result revealed that there was variation in the soil moisture storage across different places in Nigeria in the years. It could be inferred from Figure 3 that most of the places North of latitude $9^{\circ} \mathrm{N}$ recorded significantly low moisture storage below $10 \mathrm{~mm}$ in most of the months in 1983. Among the places that experienced low soil moisture storage below $10 \mathrm{~mm}$ in most of the months in 1983 included Nguru, Katsina, Kano, Maiduguri whereas Yelwa. Ngru recorded the lowest monthly soil moisture storage of between 0 and $5 \mathrm{~mm}$ in most of the months. It recorded high soil moisture storage of $122 \mathrm{~mm}, 60 \mathrm{~mm}$ and $31 \mathrm{~mm}$ in January, February and March respectively in 1983. This high record of soil moisture storage between January and March was not because of rainfall rather, because of low potential evapotranspiration and low accumulated potential water loss (APWL). It meant less water was being taking away from the soil into the atmosphere during those months. As a result of that, more water was reserved in the soil compared to other months when the amount of PE was higher to take out more water from the soil (see Tables 1a and 1b). It could be observed that stations like Jos and Kaduna recorded higher soil moisture storage of between $200 \mathrm{~mm}$ and $250 \mathrm{~mm}$ (90\%-100\% saturation) from the months of July to September. 

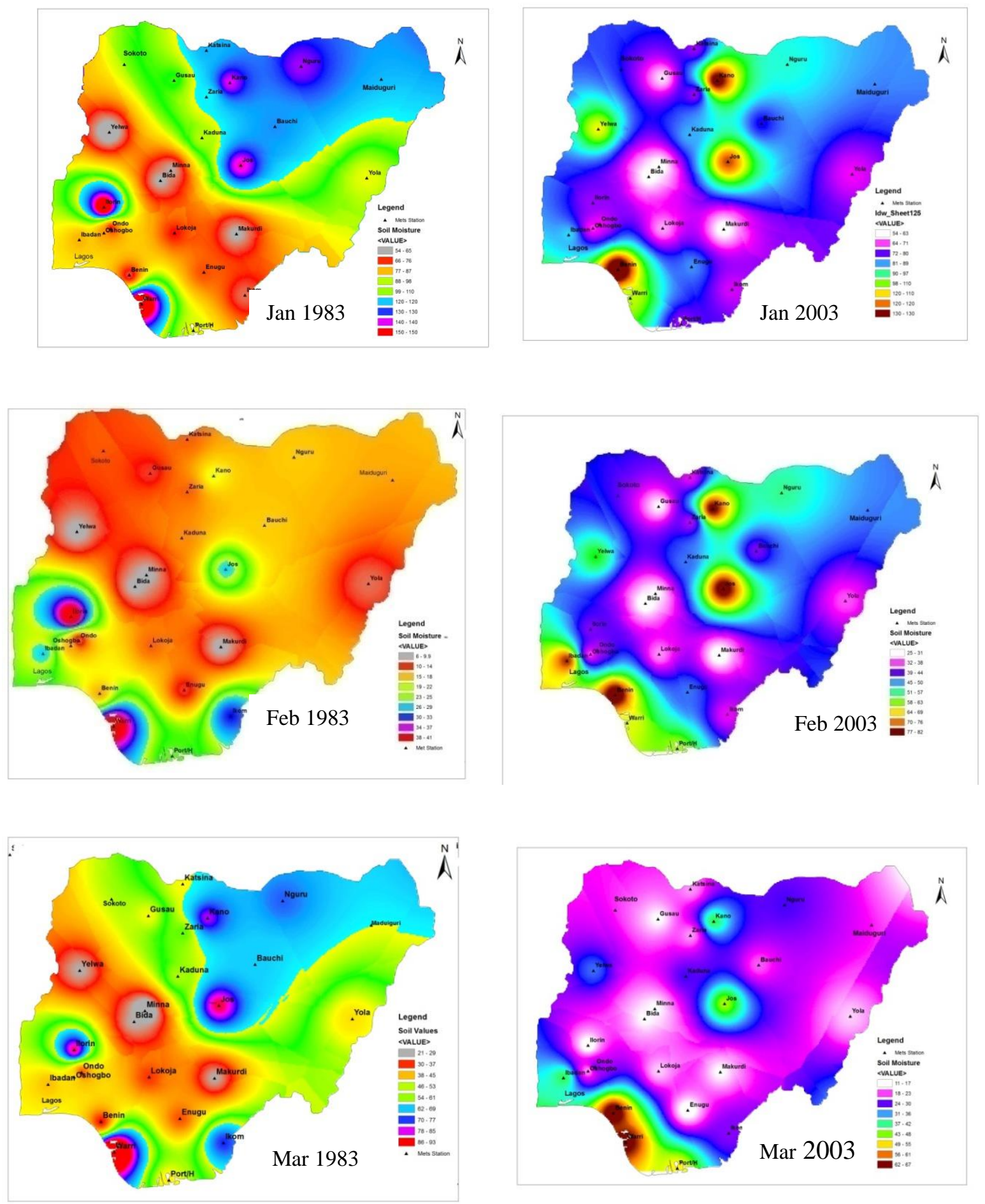

Figure 3. Spatial Pattern of monthly values of Soil Moisture Storage in Nigeria for the dry year (1983) and wet year (2003) 

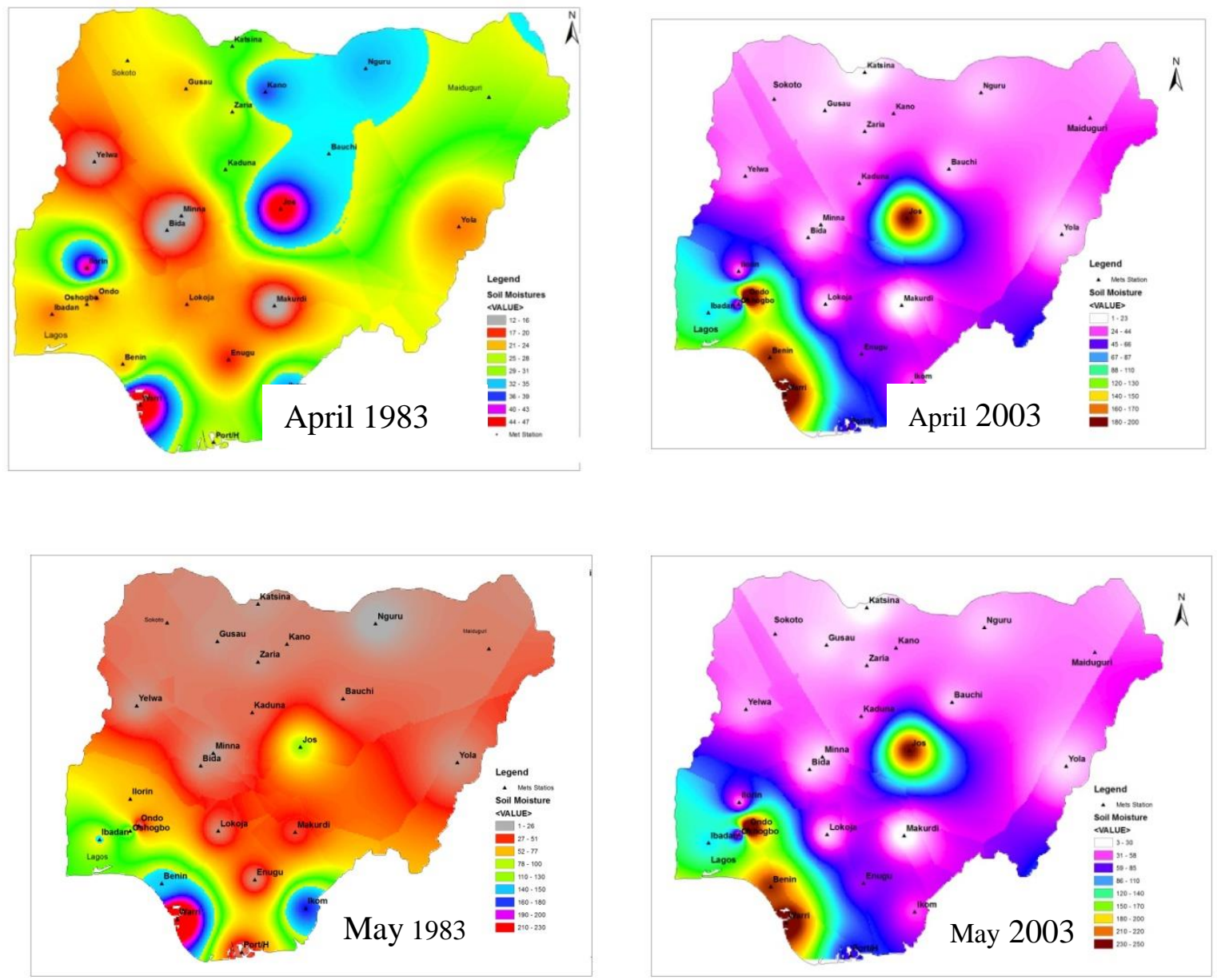

Figure 3 (continued). Spatial Pattern of monthly values of Soil Moisture Storage in Nigeria for the dry year (1983) and wet year (2003)

The result in Figure 3 also indicates that places north of latitude $9^{\circ} \mathrm{N}$ recorded low soil moisture storage between $0 \mathrm{~mm}$ to $50 \mathrm{~mm}$ between January and May in 2003 with the values of ST a bit higher in January than that of February, March, April and May. On the contrary, placesSouth of latitude $9^{\circ} \mathrm{N}$ recorded higher amount of soil moisture ranging from $100 \mathrm{~mm}$ to $250 \mathrm{~mm}$ especially between the month of July and October compared to their counterparts North of latitude $9^{\circ} \mathrm{N}$ in 2003. Notable among the places with higher soil moisture storage of $250 \mathrm{~mm}$ (100\% saturation) in four to five months in the south of latitude $9^{\circ} \mathrm{N}$ include Ikom, Calabar, Ilorin, Warri and Benin. The higher soil moisture storage recorded in the Southern part of the country may not be unconnected with the higher amount of rainfall, lower amount of potential evapotranspiration and lower accumulated potential water loss (APWL) (Tables 2a, 2b and 2c). The result in Figure 3 indicate that contrary to the year 1983 , many places in the north of latitude $9^{\circ} \mathrm{N}$ recorded higher 
soil moisture storage between $200 \mathrm{~mm}$ and $250 \mathrm{~mm}$ between the months of July and September. Locations such as: Jos, Minna, Zaria, Yelwa, Kano, Kaduna, Bauchi and Gusau north of latitude $9^{\circ} \mathrm{N}$ recorded higher monthly soil moisture storage between $200 \mathrm{~mm}$ and $250 \mathrm{~mm}$ between the month of July and September in 2003 (Figure 3). Similar to what was obtained in 1983; Ngururecorded the lowest soil moisture storage of less than $200 \mathrm{~mm}$ even during the months of July to September when the soil of other place has attained $100 \%$ saturation $(250 \mathrm{~mm})$. The result in Figure 3 further reveal that most of the places south of latitude $9^{\circ} \mathrm{N}$ recorded significantly higher soil moisture storage between $200 \mathrm{~mm}$ and $250 \mathrm{~mm}$ in most of the months compared to their counterpart north of latitude $9^{\circ} \mathrm{N}$.
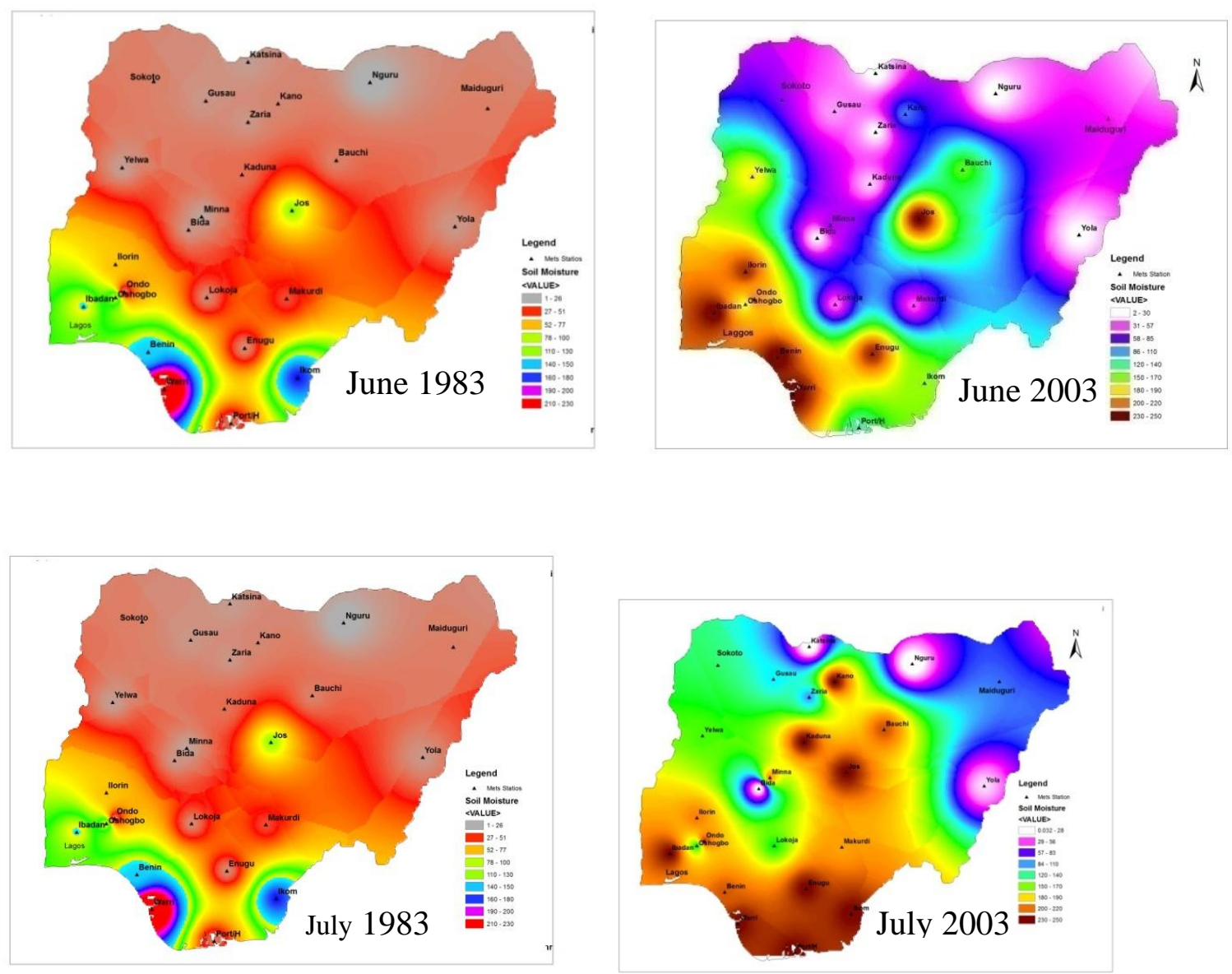

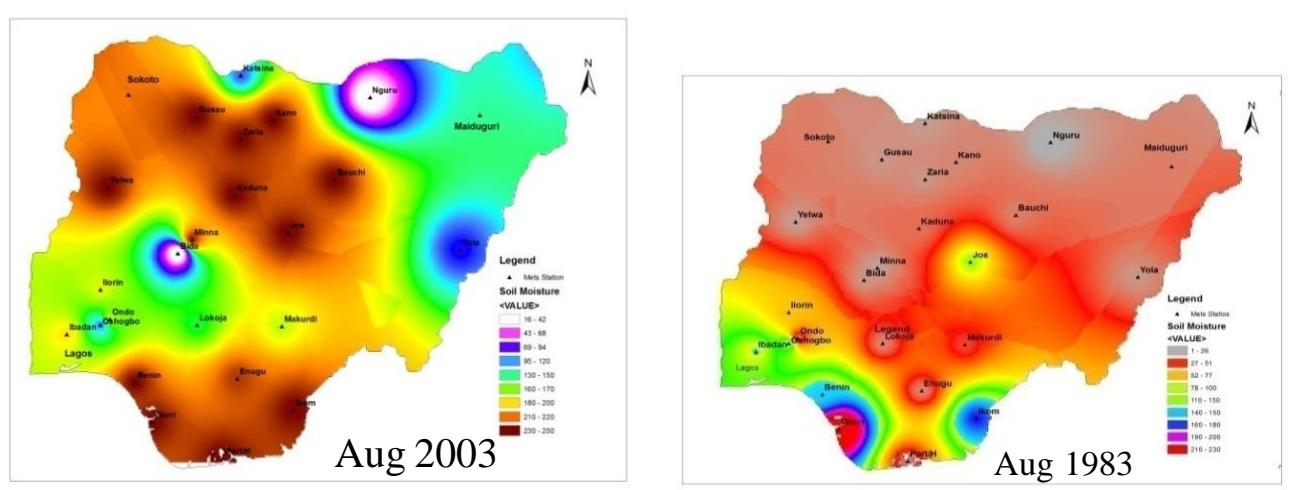

Figure 3 (continued). Spatial Pattern of monthly values of Soil Moisture Storage in Nigeria for the dry year (1983) and wet year (2003)
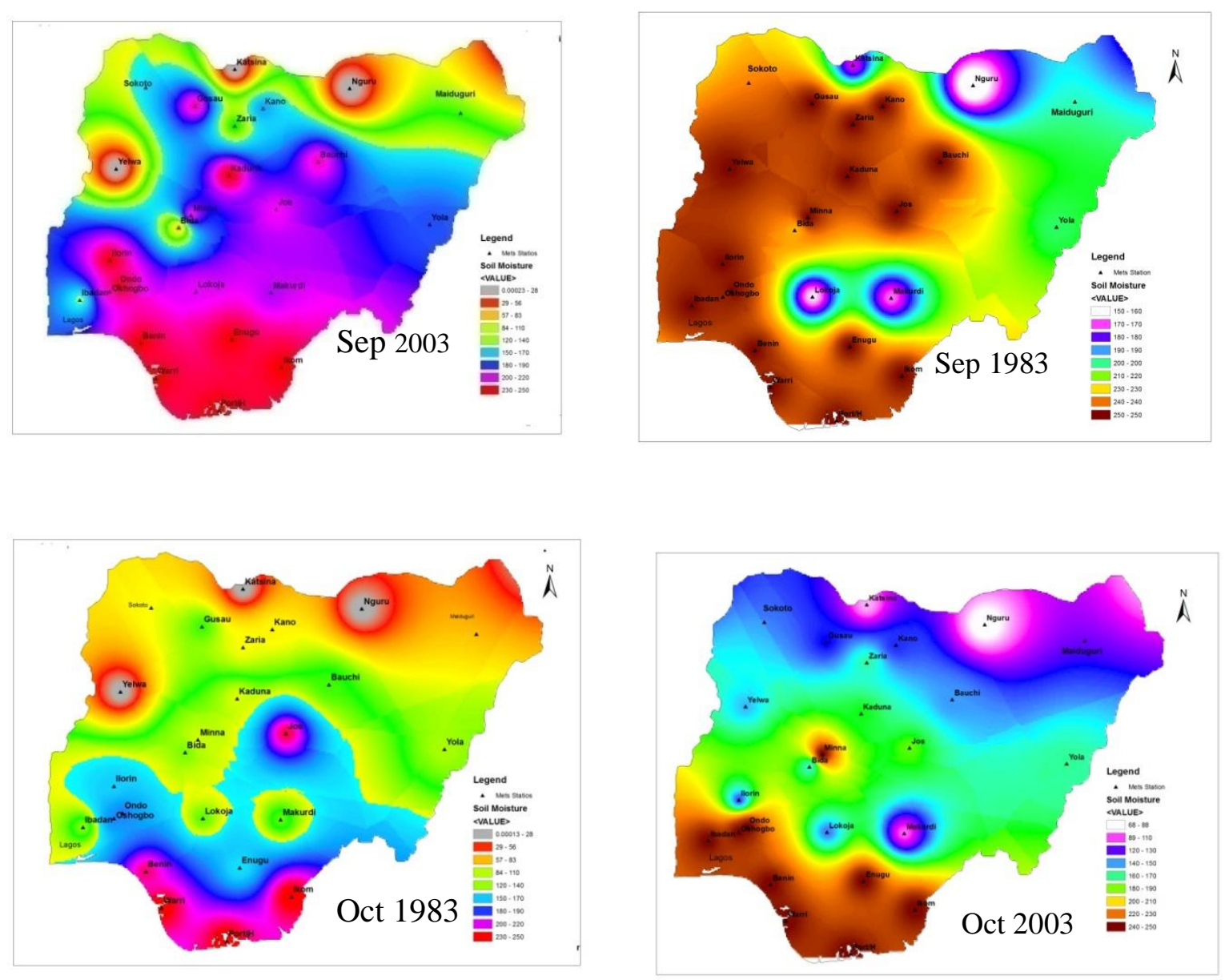

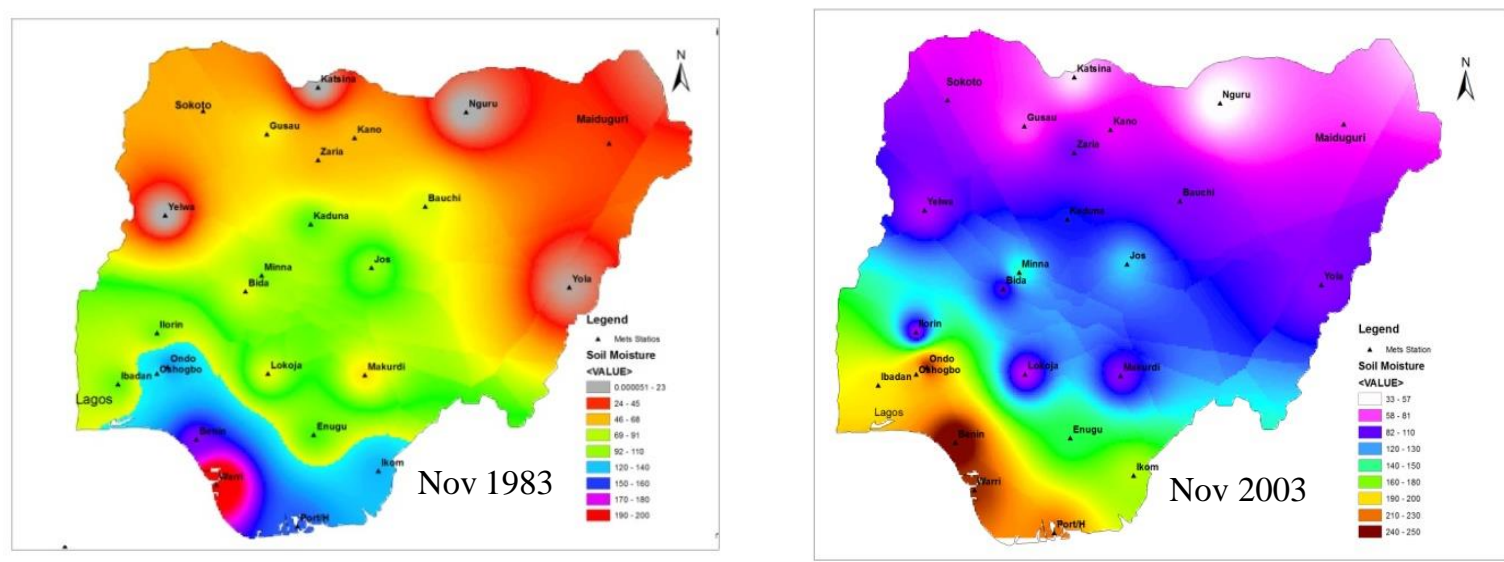

Figure 3 (continued). Spatial Pattern of monthly values of Soil Moisture Storage in Nigeria for the dry year (1983) and wet year (2003)
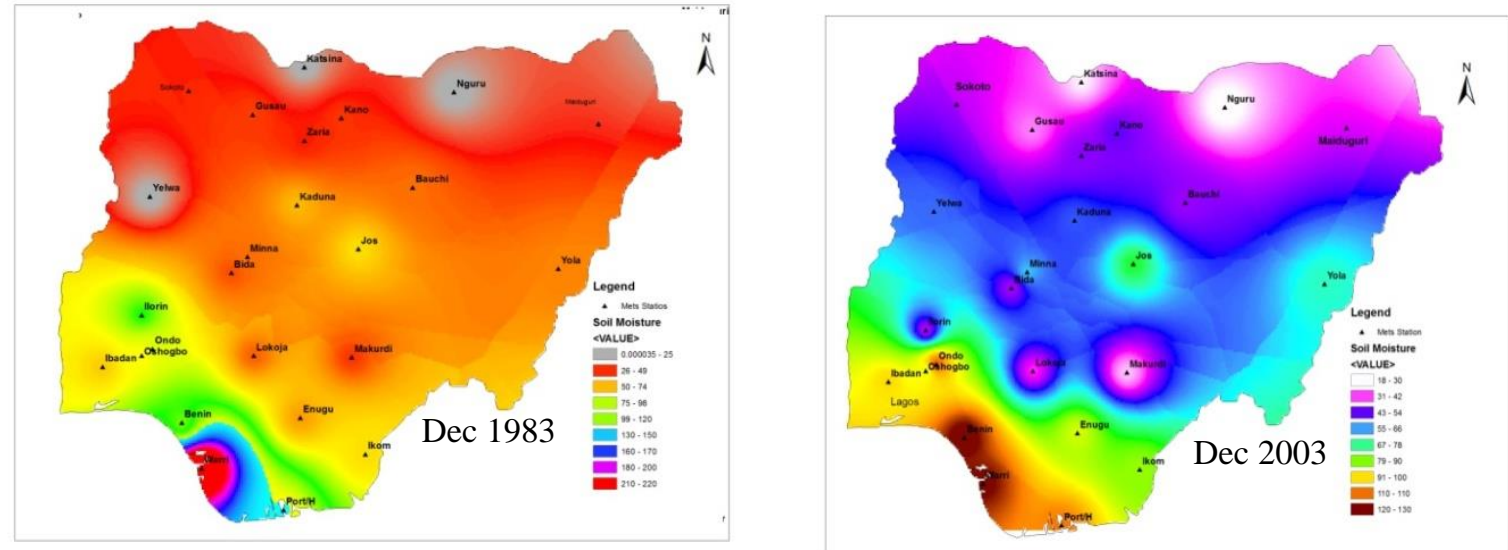

Figure 3 (continued). Spatial Pattern of monthly values of Soil Moisture Storage in Nigeria for the dry year (1983) and wet year (2003)

The factors responsible for the high soil moisture storage in Jos and Kaduna located north of latitude $9^{\circ} \mathrm{N}$ may be attributable to the high rainfall and low potential evapotranspiration recorded in those places. Jos is located on the plateau and the influence of the relief makes the temperature of the place always low compared to her counterparts within north of latitude $9^{\circ} \mathrm{N}$. It is very important to note that the amount of temperature in a place plays a significant role in the rate of $\mathrm{PE}$ of that particular place. When the temperature is high, the PE is also expected to be high and vice versa. The low soil moisture storage in most of the places North of latitude $9^{\circ} \mathrm{N}$ could be ascribed to the little or no rainfall, high amount of potential evapotranspiration and high Accumulated Potential Water Loss (APWL). The higher the amount of APWL, the lower the 
amount of soil moisture storage and the lower the amount of APWL, the higher the soil moisture storage (Tables 1a 1b; 1c; 2a, 2b and 2c). This could be the reason why most of the places recorded high soil moisture storage especially during the months of January and February (Figure 3). The implication of the low soil moisture storage recorded in some of the places across the country is an indication of insufficient water for crop production and other fauna activities (Aina et al. 2007) Even if crop manages to germinate under insufficient soil moisture condition, it may likely withers after longer days of soil moisture deficit. Likewise, some organisms cannot survive under low soil moisture storage. Rather, they survive when the soil has attained reasonable amount of moisture storage for their survival e.g earthworm and some other amphibians (Tyler, 2017)

It is equally crucial to note that, the higher soil moisture storage recorded in most of the months signify sufficient water for crop production. Soil moisture storage does not only influence crop growth. Rather, it also influences the micro and the boundary layer climate (Oke, 1987). If there is adequate water in the soil, the incoming solar radiation will be used for convective activities. On the contrary, if the moisture is very low or totally in deficit on the earth's surface, the incoming solar radiation will only be used to warm the environment thereby bringing physiological discomfort (Ayoade, 1995). The more water in the soil, the more slowly the soil gets heated during the daytime and the less during the night (Zachary, 2013 and Oke,1987). The less the water in the soil, the faster the soil gets heated during the daytime and the faster it loses the heat. The computed values of soil moisture storage give an indication of the pattern of soil moisture profile across different locations in Nigeria. This is vital information to assessing the risk of flooding in an area given the input precipitation (Ayoade, 1995). Any additional input precipitation after the soil has attained its storage capacity runs off either to a nearby river or a stream. If runoff could not get its way through a proper drainage channel, it may lead to flooding. An incident that can claim many lives and properties. The spatial pattern of soil moisture storage across different locations in Nigeria is a panacea to identify places that deserve irrigation schemes to boost agriculture production.

\section{Temporal Pattern of Soil Moisture Storage in Nigeria}

The result indicates that many places across Nigeria recorded very low soil moisture storage especially during the months of March, April and May in both 1983 and 2003 in Nigeria. It is important to note that most places across Nigeria recorded high soil moisture storage between 
$50 \mathrm{~mm}$ and 150mm during the months of January and February in both 1983 and 2003. This is not because of the input precipitation but rather, due to the influence of the harmattan wind, which significantly reduces the amount of potential evapotranspiration (see Tables $1 \mathrm{a}$ and $1 \mathrm{~b} ; 2 \mathrm{a}$ and $2 \mathrm{~b}$ ). The values of monthly soil moisture storage for individual location in Nigeria are shown in Figure 3. It could be observed from Figure 4 that most of the stations recorded low soil moisture storage of between 2mm and 20mm from the month of February to May in both 1983 and 2003 especially in the North. In fact, some locations recorded less than $2 \mathrm{~mm}$ soil moisture storage even during the months of July and September when the soil moisture of many locations have attained $250 \mathrm{~mm}$ (100\% saturation) in 1983. It is evident from Figure 3 that the soil moisture increases from less than $100 \mathrm{~mm}$ to as high as $200 \mathrm{~mm}$ and above in most of the locations during the months of July, August, September and October in both 1983 and 2003. It could be observed from Figure 4 that the highest soil moisture storage was recorded during the month of September. The soil moisture storage of virtually all places across the country reached their full storage capacity of $250 \mathrm{~mm}$ (100\% saturation) during the month of September. This is followed by the months of August, October and July with moisture storage of between 200mm and 250mm in both 1983 and 2003. 

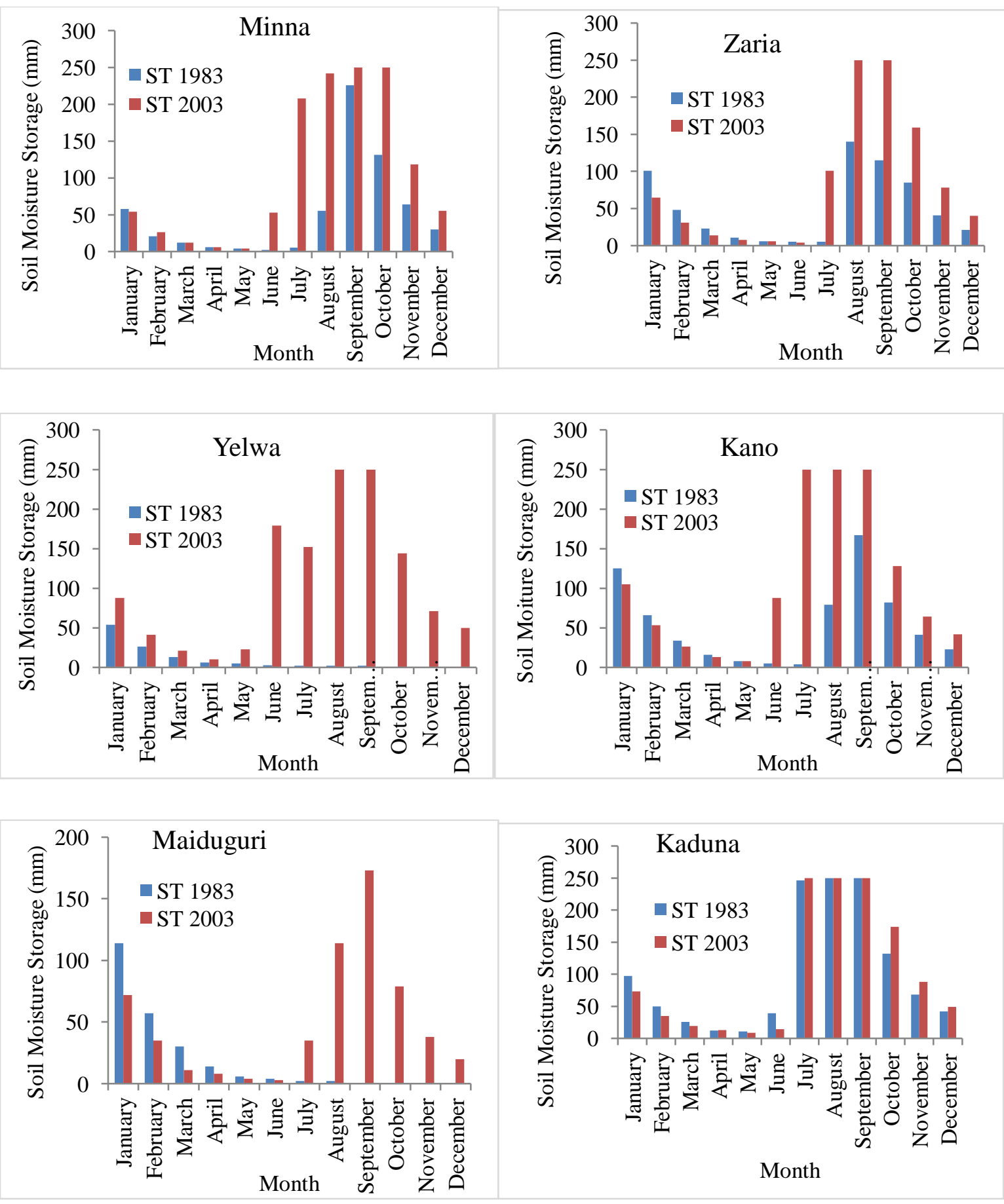

Figure 4. Monthly Variation of Soil Moisture Storage for the Individual Locations in Nigeria for 1983 and 2003

Source: Author's Computation 


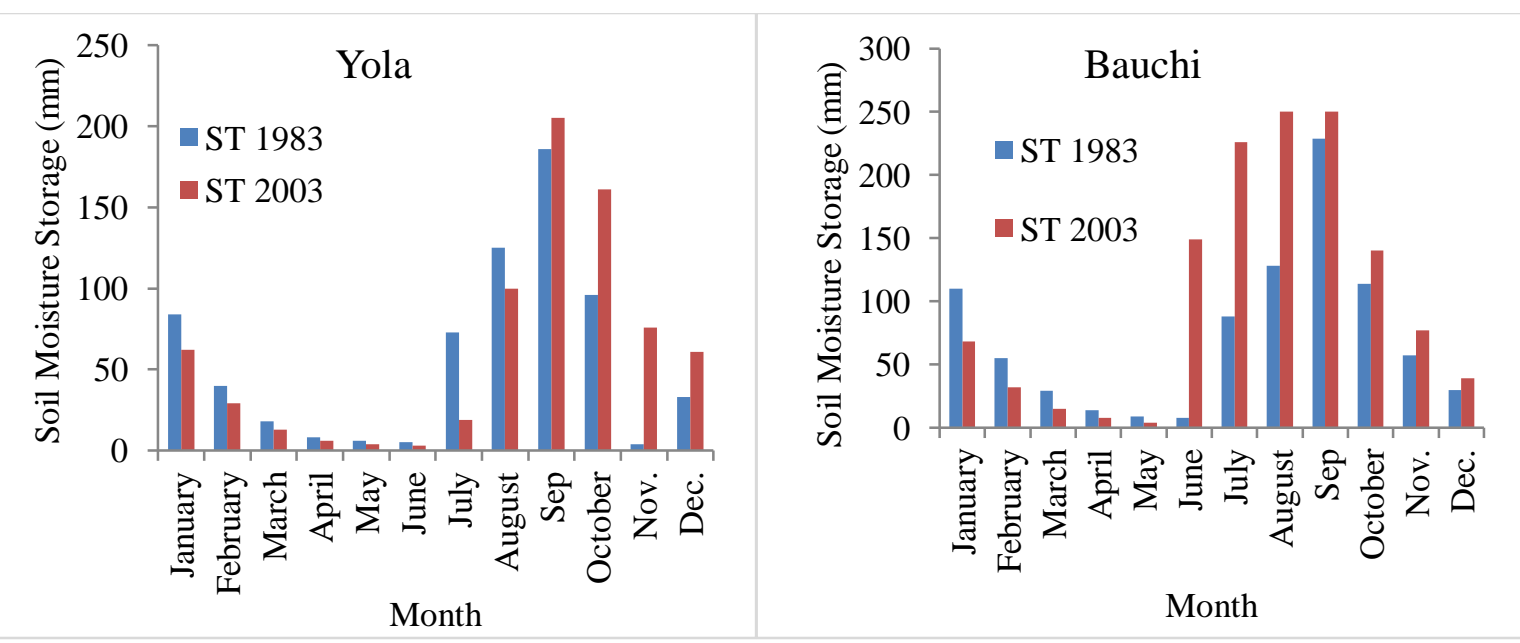

Figure 4. Monthly Variation of Soil Moisture Storage for the Individual Locations in Nigeria for 1983 and 2003. Source: Author's Computation

This monthly variations in soil moisture storage indicate the insufficient moisture in the soil for crop growth in most places in Nigeria from the months of January to April as well as October to December. The values of soil moisture storage across the country also indicate the commencement of growing season to be around April in most part of the Southern part of Nigeria and around June/July in most part of the North (see Figures 4). It is also important to note that the amount of moisture in the soil begins to decline from above $100 \mathrm{~mm}$ in October to less than $60 \mathrm{~mm}$ and as low as below 40mm between November and December in most places both 1983 and 2003. The values of monthly soil moisture storage across different locations in Nigeria make it possible to identify the months of July, August and September as the flood risk months in Nigeria. This is because virtually all places in Nigeria attained $100 \%$ moisture storage capacity. This information, if properly utilized can assist to minimize the incidences of flooding in Nigeria. It is evident that most locations across the country recorded soil moisture storage of above $50 \mathrm{~mm}$ in the months of January in 1983 and 2003 (see Figure 4). This could be because of the low PE and APWL, which is an influence of the harmattan wind. The high soil moisture storage is not because there is high record of rainfall during the months of January, February and March but because there is low potential evapotranspiration during the months. The climatic water budgeting method assumes even if there is no rainfall, evapotranspiration will continue. The rate of PE is always low during the cool months. As such, the low PE will reduce the amount of water loss and more water in the soil. On the other hand, the rate of PE is always high during the warm months and this increases the rate and amount of water loss from the soil (Moreland, 1993). The monthly values of soil 
moisture storage at different locations in Nigeria will serve as a useful guide in understanding the level of soil moisture storage at different locations and at different time of the year in Nigeria. The temporal pattern of soil moisture storage in Nigeria will enable the farmers to plan the best time to commence the growing of their crops and to understand cessation of the growing season. The temporal pattern of the soil moisture in Nigeria as revealed by this study will not only be useful to agriculture rather; but alsoto the stakeholders in water resources management to design and forecast the best water management strategies. This will be crucial in $=$ forestalling incidence of flooding especially during the months in which the soil of many places across Nigeria might have reached their water holding capacity. Those months with low soil moisture storage indicate the need for irrigation scheme across many places in Nigeria to facilitate all year round farming in. 

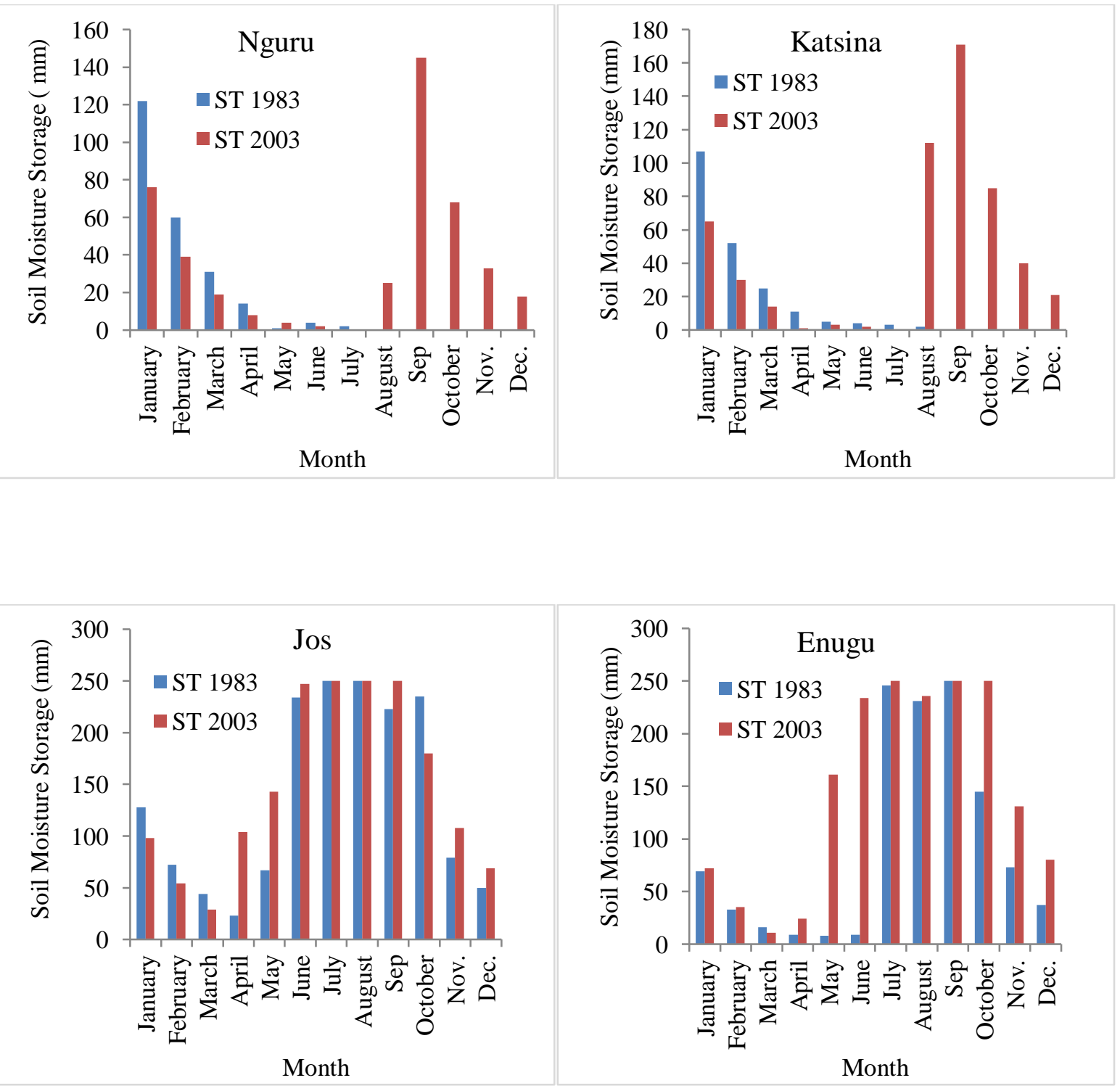

Figure 4 (continued). Monthly Variation of Soil Moisture Storage for the Individual Locations in Nigeria for 1983 and 2003 

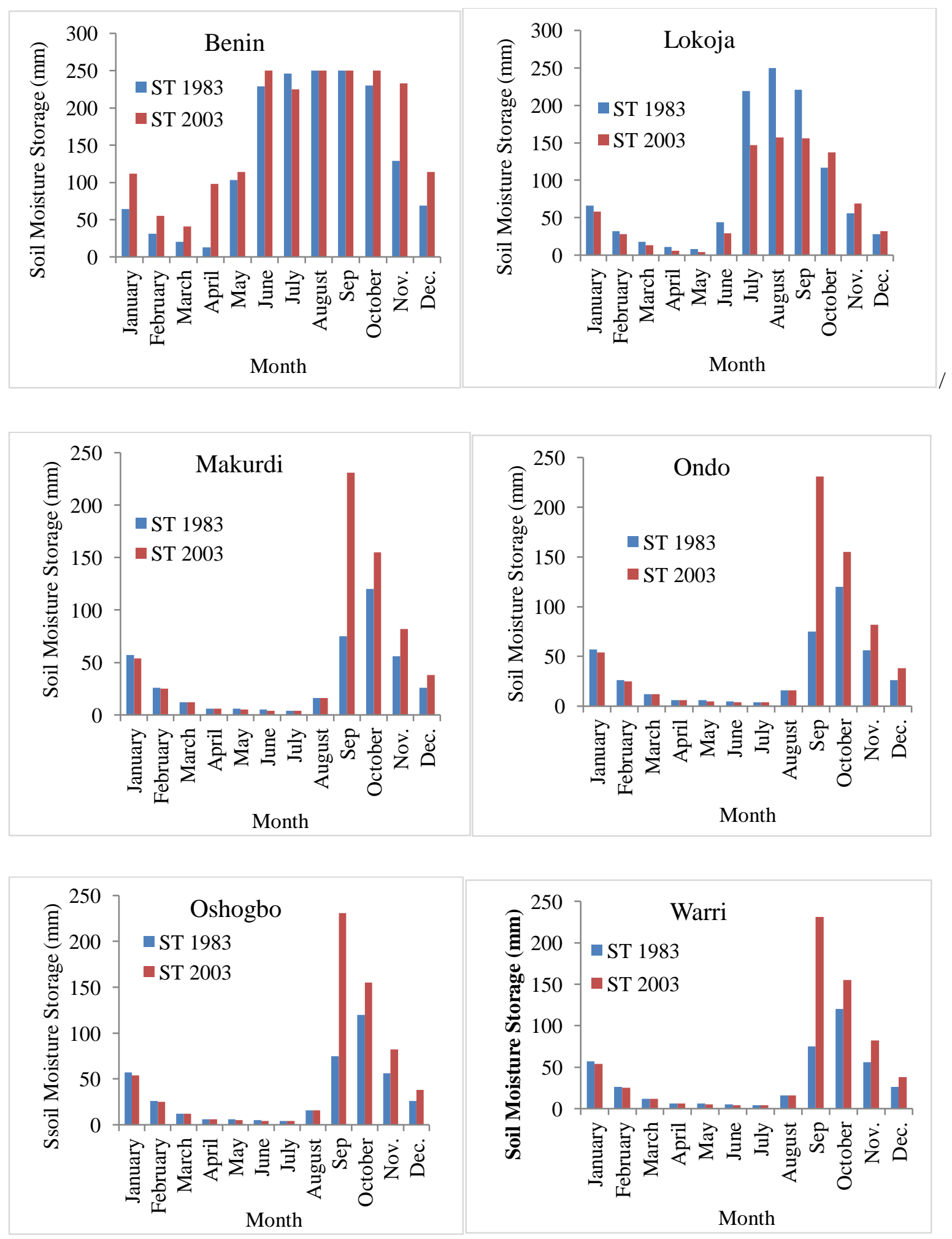

Figure 4 (continued). Monthly Variation of Soil Moisture Storage for the Individual Locations in Nigeria for 1983 and 2003 

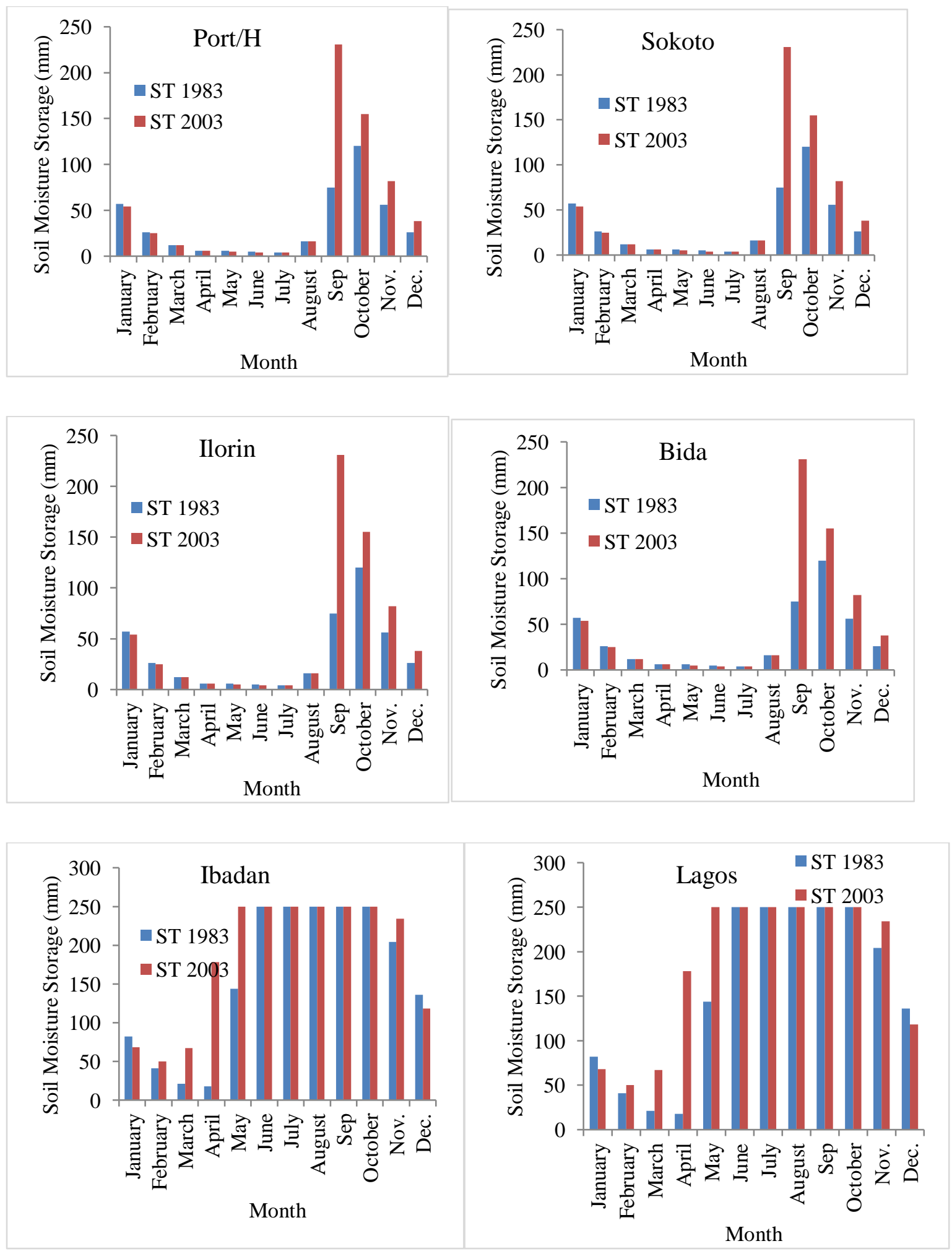

Figure 4 (continued). Monthly Variation of Soil Moisture Storage for the Individual Locations in Nigeria for 1983 and 2003 

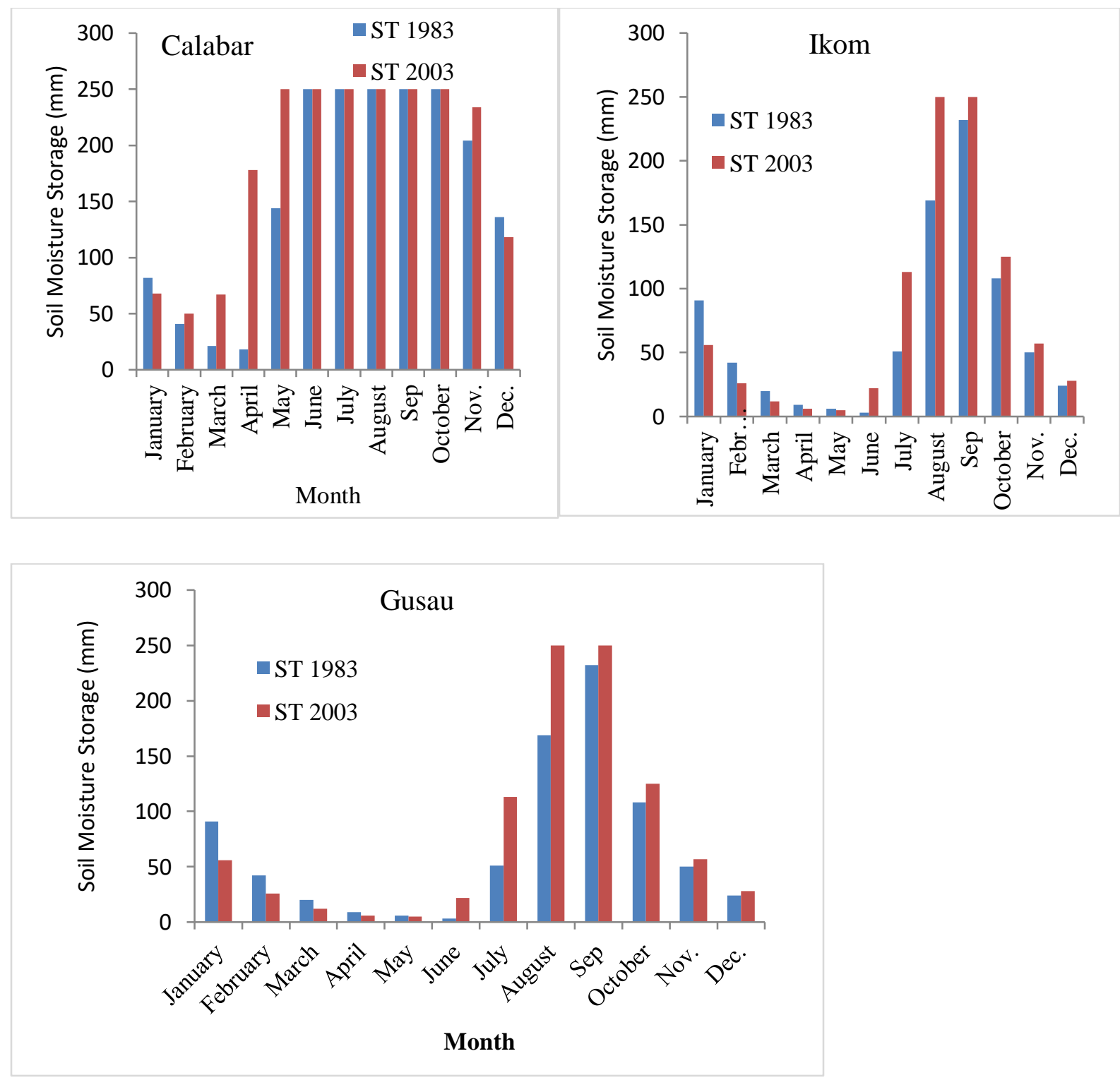

Figure 4 (continued). Monthly Variation of Soil Moisture Storage for the Individual Locations in Nigeria for 1983 and 2003

\section{Variation between the Soil Moisture Storage of 1983 and 2003 in Nigeria}

The results in Figure 3 show that there is variation between the soil moisture storage of 1983 and 2003 in Nigeria. The numbers of locations that attained soil moisture storage of $250 \mathrm{~mm}$ in 2003 during the months of July, August and September are many compared to 1983. It is only in Kaduna and Jos states in the North of latitude $9^{\circ} \mathrm{N}$ that soil moisture storage attained $250 \mathrm{~mm}(100 \%)$ in 1983. The soil moisture storage for 1983 and 2003 were subjected to pair t test and the result in Table 3 and Table 4 revealed that there is a significant difference between the soil moisture storage 
for 1983 and 2003 in Nigeria. With reference to Tables 3 and4, .000 is less than $\propto(0.05)$. Therefore, "paired sample t test reveals statistical difference between the soil moisture storage of $2003(\mathrm{M}=1299.56, \mathrm{~s}=515.45)$ and $1983(\mathrm{M}=1001.56, \mathrm{~S}=551.66)$ in Nigeria. $\mathrm{t}(26)=4.420, \mathrm{P}=.000$, $\propto 0.05$. This means there is a significant difference in the soil moisture storage of 2003 and that of 1983 in Nigeria. The low soil moisture storage recorded in 1983 compared to 2003 in Nigeria could be attributed to the fact that the decade 1980s was bedeviled with drought of varied magnitudes. The year 2003 on the other hand, recorded high soil moisture storage across many locations in Nigeria because this year is described as a recovery year in Nigeria when there was more rainfall (Umar, 2013). This finding is in agreement with Kehinde (2017) which revealed that there is a significant different between the water balance indices of 1983 and 2003 and across different locations in Nigeria.

Table 3. Paired Sample Statistics

\begin{tabular}{llllll}
\hline & & Mean & N & Std. Deviation & $\begin{array}{l}\text { Std. Error } \\
\text { Mean }\end{array}$ \\
\hline \multirow{2}{*}{ Pair 1 } & ST 2003 & 1299.56 & 27 & 515.44 & 99.19 \\
& ST 1983 & 1001.56 & 27 & 551.66 & 106.17 \\
\hline
\end{tabular}

Source: Author's Computation

Table 4. Paired Samples Test

\begin{tabular}{|c|c|c|c|c|c|c|c|c|c|}
\hline & & \multicolumn{5}{|c|}{ Paired Differences } & \multirow{3}{*}{$\mathrm{t}$} & \multirow{3}{*}{ df } & \multirow{3}{*}{$\begin{array}{l}\text { Sig. } \\
\text { tailed) }\end{array}$} \\
\hline & & \multirow[t]{2}{*}{ Mean } & \multirow[t]{2}{*}{$\begin{array}{l}\text { Std. } \\
\text { Deviation }\end{array}$} & \multirow{2}{*}{$\begin{array}{l}\text { Std. } \\
\text { Error } \\
\text { Mean }\end{array}$} & $\begin{array}{l}\text { Interva } \\
\text { Differe }\end{array}$ & of the & & & \\
\hline & & & & & Lower & Upper & & & \\
\hline $\begin{array}{l}\text { Pair } \\
1\end{array}$ & $\begin{array}{l}\text { ST } \\
\text { 2003- } \\
\text { ST1983 }\end{array}$ & 298 & 350.36 & 67.43 & 159.4 & 436.59 & 4.42 & 26 & 0 \\
\hline
\end{tabular}

Source: Author's Computation 


\section{Conclusion and recommendations}

The study assessed the monthly soil moisture storage at different locations in Nigeria for 1983 and 2003. Based on the findings available, I concluded that soil moisture storage varies spatiotemporally and that the variations are function of the amount of the rainfall, potential evapotranspiration (PE) and the Accumulated Potential Water loss (APWL). The soil moisture storage increases from the north to the south. While the amount of soil moisture storage was very low from March to June in both years especially in the northern part of Nigeria, it was very high from July to September. The study suggests the need for more irrigation projects across Nigeria and expansion of the capacity of the existing ones to be more productive for efficient food productions across Nigeria. Government should also intensify efforts to create awareness about the likely incident of flood from the months of July to September when the soil moisture of different places across the country must have attained their full storage capacity in order to take proactive measures against flood occurrence. Irrigation agriculture should not be limited to only the Northern part of Nigeria. Rather, it should be extended to some other locations in the Southern part of Nigeria as well, because of rainfall variability and dry spells which truncate the length of the growing season in Nigeria.

\section{References}

Aboyade, O. (1990). Some Missing Policy Links in Nigeria. Agricultural Development. International. Institute of Tropical, Agriculture (IITA) University Press, Ibadan, Nigeria.

Ahmad, S., \& Hashim, N. M. (2007). Effects of Soil Moisture on Urban Heat Island Occurrences: Case of Selangor, Malaysia. Humanity Social Science Journal, 2: 132-138

Aina, O. O., Dixon, A. G. O. and Akinrinde, E. A. (2007). Effect of Soil Moisture Stress on Growth and Yield of Cassava in Nigeria. Pakistan Journal of Biological Science, 10: 3085-3090. https://doi.org/19090103

Andrew, N. G, Thomas, A. S, and Mark, J. E. (2002). Microclimate and Soil Moisture Resources to Gap Information in Coastal Douglas-fir Forests. Canadian Journal of Forest Research, 32 (2): $332-343$. 
Ati, O.F., Stigter, C. J., Oladepo, E. O. (2001). A Comparison of Methods to Determine the Onset of the Growing Season in Northern Nigeria, International Journal of Climatology, (22): 731742. 002ijoc.712https://doi.org/10.1.

Ati, F. O. (1996). Comparison of methods to detect the onset of growing season and its trends for some Stations in the Sudan Savanna of Northern Nigeria. (Unpublished Master's Thesis). Department of Geography, Ahmadu Bello University, Zaria, Nigeria.

Ayoade, J. O. (1995). Climate and human welfare. Inaugural lecture delivered at the University of Ibadan (Pp: 6-23). Published by Ibadan University Press.

Ayoade, J. O. (1995). Climate and human welfare. Inaugural lecture delivered at the University of Ibadan (Pp: 6-23). Published by Ibadan University Press.

Ceri, E.E. and John, R. E. (1999). The Effect of Soil Water Potential on Seedling Growth of Some British Plants. New Phytol. (1991), 118, 571-579.

Chukwudi, P. N, Emma, E. E, Ifeanyi, C. E and Nwabueze, I. I. (2017). Analysis of Trends in Rainfall and Water Balance Characteristics of Awkwa, Nigeria. Journal of Geography and Regional Planning, 10 (7): 186-196. https://doi: 10.5897/JGRP2016.0603

Copernicus, (2013). Soil moisture condition; the European earth observation programme. Retrieved May 15, 2015 from http//www.copernicus.edu/page.principal/library.com.

Fabura, M. I. (2005). Scheduling irrigation in an area transmitting from rain-fed to irrigated agriculture Using Water Approach. Proceedings of the $6^{\text {th }}$ International Conference of the Nigerian Institution of Agriculture Engineers, Yenogoa, 27:95-108.

Fasinmirin, J. T., and Olufayo, A. A. (2009). Yield and Water Use Efficiency of Jute Mallow Corchorus Olitorius Varying Soil Water Management Strategies. Journal of Medical Plants, 3 (4): 186-191. https://www.academicjournals.org/JMPR.

Fasinmirin, J. T., and Oguntuase, A. M. (2008). Soil Moisture Distribution Pattern in Amaranthus Cruentus Field under Drip Irrigation System. African Journal of Agricultural Research, 3 (7): 486-493. https://www.academicjournals.com

Houser, S. and Asawalam, D. O., (1998). Effects of Fallow System and Cropping Frequency Upon Quantity and Composition of Earthworm Casts. Int. Inst. Tropical agric. Humid forest station, BP 2008 (Messa) Yaounde, Cameroon. https://alm.engr.edu/cb/wiki/17027

Iwena, O. A. (2000). Essential Geography for senior secondary schools. Tonal Publishers LTD Ikeja Lagos, Nigeria. Pp: 231-250. 
Isoteim, F. M. (2014). Mathematical Relationship between Soil Moisture and Ground Water Level in a Loamy Sandy Soil in the Niger Delta Region of Nigeria. International Journal of Advanced Research in Engineering and Technology (IJARET), 5 (12):18.https://www.researchgate.net/publication/26990538

International Water Management Institute (IWMI) (2011). Retrieved November, 2017 from www.iwmi.cgiar.org/about/soil/overview/ International Water Management Institute.

James, E. A. (1999). Soil moisture. Retrieved November 24, 2017 from https//weather.msfc.nasa.geov/landprocess/.

Jose Ricardo, M. P, Cristiam, B, Maria Luiza, F. N, Paricia, M. S \& Renan, S. P (2015). Microclimate and Soil Moisture in a Silvopastral System in Southeastern Brazil. Bragantia, 74 (1) https://dx.doi.org/10.1590/1678-4499.0334

Kehinde, M.O, Umar, A. T, Bulama, L (2019) Relationship between Accumulated Potential Water Loss and Soil Moisture Storage: A Case Study of Sudano-Sahelian Region of Nigeria. Proceedings of the Nigerian Meteorological Society 2019 International Conference on Climate Change Challenges and Prospects (NMetS 2019

Kehinde, M. O. (2017). Assessment of spatio-temporal pattern of some water balance indices in Nigeria (Master's Dissertation). Department of Geography, Usmanu Danfodiyo University Sokoto, Nigeria. Published by Lap LAMBERT Academic Publishing, Germany. Pp: 1-217.

Kirien, W \& Sonia, I. S. (2015). Impact of Soil Moisture on Extreme Maximum Temperature in Europe. Journal of Science, 9: 57-67.https://doi.org/10.10616

Kumar, P. U. (1995). Estimation of Ground Water Recharge Using Soil Moisture Balance Approach. Journal of Indian Water Resource Society, 1 (2): 1116.https://www.researchgate,net/publication/215973823

Lunt, I. A, Hubbard, S. S., and Rubin, Y (2004). Soil Moisture Content Estimation Using Ground Penetration Radar Reflection Data. Journal of Hydrology, 307 (2005): 254269.https://wenku.baidu.com

Maraghni, M. and Neffati, M. (2010). Seed Germination at different Temperature and Water Stress Levels and Seedling Emergence from Different depths of Ziziphus Lotus. South African journal of Botany, 76 (3), 453-459. https://doi.org/10.1016/j.sajb.2010.02.092 .

Moreland, J. A. (1993). Drought: U.S. geological survey water sheet, Open-File Report 93-642, $2 p$. 
Ngetich, K. F and Mugendi, D. N (2014). Length of Growing Season, Rainfall Temporal Distribution, Onset and Cessation Dates in Kenya Highlands. Agriculture and Forest Meteorology, 188: 24-32 https://doi.org/10.1016/.

Odunze, A. C., Kudi, T. M., Daudu, C., Adeosun, J., Ayoola, G., Amapu, I. Y., Abu, S. T., Mand, A., Ezul, G., and Constance, D (2010) Soil Moisture Stress Mitigation for Sustainable Upland Rice Production in the Northern Guinea Savanna of Nigeria. Agriculture and Biology Journal of North America. 2651-7525.https://doi.org/10.525.

Oke, T. R. (1987). Boundary layer climate, second edition. Methuen \& Co. in association with Methuen, Inc. New York. Pp: 1-60.

Rita, N (2007). Determination of Length of Growing Season in Samaru Using Different Potential Evapotranspiration Models. AU. J. T., 11 (1): 28-35.

Ritter, M. E. (2012). The Physical Environment: An Introduction to Physical Geography. Retrieved April 12, 2017, from http//www.earthonlinemedia.com/ebooks/tpe-3e/tittlepage.html

Shiwachi, H, Komoda, M, Koshio, K andTakashi, H. (2008). Effect of Soil moisture Stress on the Growth of Crchorus Olitorius L. African Journal of Agricultural Research, 4 (4): 289-293.

Syed, Z. H., Stephane, B., and Sylvie, L (2013) Influence of Soil Moisture on Urban Microclimate and Surface-Layer Meteorology in Oklahoma City. Journal of Applied Meteorology and Climatology, 53: 83-98.https://doi.org/10.1175/JAMC-D-13-0156.1.

Thornthwaite, C. W. (1948). "An Approach towards a Rational Classification of Climate" Geographical Review, 38(1): 55-94.https://doi.org/10.2307/210739.

Thornthwaite Monthly Water Balance Model. (2020). In Wikipedia. Retrieved on 17 September 2020 from https://alm.engr.edu/cb/wiki/1702

Timbale, B., Power, S., Colman, R., Viviand, J., \& Lirola, S. (2002) “Does Soil Moisture Influence Climate Variability and Predictability over Australia?" Journal of Climate, (15): 12301238.https://doi.org/10.1175/1520-0442.

Tyler, L., (2017). Factors that affect the growth of microorganisms. Retrieved January 4, 2018 from https://sciencing.com/factors-affecting-growth-microorganisms-5299917.html.

Ufoegbune, G. C, (2016). Effect of Climate Change on the Onset and Cessation of Rainfall and Length of Growing Season of selected Crop in the Mangroove Savanna and Transition Zone in Nigeria, Jordan Journal of Agricultural Science, 12 (2). 
Umar A. T. (2013). Frequency and Spatial Pattern of Droughts in Nigeria. Journal of Meteorology \& Climate Science, 11 (1): 58-74.

Umar, A. T. (2010). Recent Trend and Variability in the Length of Growing Season in Northern Nigeria. Journal of Meteorology and Climate Science, 8 (1): 40-52.

Varallyay, G. (2010). The Impact of Climate Change on Soils and on their Water Management. Journal of Agronomy Research, 8 (2): 385-396. 